Artículo de posesión

\title{
Evolución del riesgo de extinción y estado actual de conservación de las aves de Colombia
}

\author{
Luis Miguel Renjifo*, Ángela María Amaya-Villarreal \\ Departamento de Ecología y Territorio, Facultad de Estudios Ambientales y Rurales, \\ Pontificia Universidad Javeriana, Bogotá, D.C., Colombia \\ Artículo de posesión para el ingreso como miembro correspondiente de la \\ Academia Colombiana de Ciencias Exactas, Físicas y Naturales el 26 de abril de 2017
}

\section{Resumen}

La evaluación periódica del riesgo de extinción de las especies es indispensable para monitorear el progreso o retroceso de su estado de conservación. Se analiza aquí la evolución de las listas y libros rojos de aves en Colombia en cuanto al sistema de evaluación, los métodos y la composición de las especies. Las cinco evaluaciones realizadas a la fecha utilizaron el sistema de la Unión Internacional para la Conservación de la Naturaleza (International Union for Conservation of Nature, UICN). Los métodos han progresado notablemente desde las evaluaciones que se basaban exclusivamente en el criterio experto hasta las actuales que utilizan herramientas analíticas sofisticadas sin dejar de usar el criterio experto. En total, 62, 81, 89, 112 y 140 especies estuvieron en alguna categoría de amenaza en los años 1986, 1998, 2001, 2002 y 2016, respectivamente. Al comparar las evaluaciones del 2002 y el 2016, la proporción de especies bajo alguna categoría de amenaza se ha reducido para el criterio A (rápido declive poblacional), es estable para el criterio B (distribución pequeña, fragmentada y en disminución), en tanto que ha aumentado para el criterio C (población pequeña y en disminución). La agricultura, la ganadería, la cacería y el tráfico ilegal, así como los cultivos ilícitos, son las amenazas directas más significativas actualmente $(55,44,32$ y $31 \%$ de especies afectadas, respectivamente). Tomadas en su conjunto, estas amenazas sugieren que la principal causa de amenaza para las aves es la debilidad del control del Estado sobre el territorio. Las familias Psittacidae, Grallariidae y Cracidae son particularmente sensibles. La precisión de futuras evaluaciones se beneficiará con la información sobre las densidades poblacionales en diferentes tipos de hábitats y gradientes de idoneidad, así como con los registros de georreferenciación de alta calidad y la exploración de regiones con escasos niveles de información ornitológica. (c) 2017. Acad. Colomb. Cienc. Ex. Fis. Nat.

Palabras clave: Evaluación de riesgo de extinción; Especies amenazadas; Aves; Colombia.

Evolution of extinction risk and current conservation status of Colombian birds

\begin{abstract}
Periodic assessment of extinction risk is fundamental to monitor progress or regression on species conservation status. We analyzed changes through time in red lists and red data books in Colombia in terms of evaluation systems, methods, and species composition. All five assessments used the International Union for Conservation of Nature (IUCN) system. Methods have progressed notably, from assessments $100 \%$ based on expert knowledge to assessments that use sophisticated analytical tools together with expert knowledge. A total of 62, 81, 89, 112 and 140 species were threatened in the years 1986, 1998, 2001, 2002 and 2016, respectively. Comparing assessments conducted in 2002 and 2016, the proportion of species in a category of threat under criterion A (rapid population decline) has declined, it is stable under criterion B (small, fragmented, and declining distribution), and has increased under criterion C (small and declining population). Agriculture, livestock farming, hunting and illegal trade, as well as illicit crops, are the most significant direct threats currently affecting $55 \%, 44 \%, 32 \%$, and $31 \%$ of the species, respectively. This set of threats taken together suggests that the main direct threat for birds is the weakness of State control inin the country's territories. The families Psittacidae, Grallariidae, and Cracidae are particularly sensitive. Future assessments will benefit with new information on population densities in different habitat types, and suitability gradients, as well as high-quality georeferencing records, and exploration of regions with low levels of ornithological knowledge. (c) 2017. Acad. Colomb. Cienc. Ex. Fis. Nat.
\end{abstract}

Key words: Extinction risk assessment; Threaten species; Birds; Colombia.

\footnotetext{
*Correspondencia:

Luis Miguel Renjifo, 1mrenjifo@javeriana.edu.co; Recibido: 29 de marzo de 2017; Aceptado: 03 de noviembre de 2017
} 


\section{Introducción}

El intenso y creciente uso de recursos naturales por parte de la humanidad afecta la biodiversidad al producir la degradación, la destrucción y la fragmentación de los hábitats, tanto como la emisión de contaminantes, la introducción de especies invasoras y la explotación excesiva de especies, lo que incluso ha llevado a la extinción de muchas de ellas (Pimm \& Raven, 2000, Foley, et al., 2005, Pereira, et al., 2010, Rands, et al., 2010, Dirzo, et al., 2014, Haddad, et al. 2015). La pérdida de especies disminuye la capacidad de los ecosistemas para mantener funciones tan importantes como la provisión de agua, de recursos forestales y de alimentos (Dirzo, et al., 2014). La pérdida de especies también tiene consecuencias evolutivas y conduce a perturbaciones en cascada que se propagan a través de los ecosistemas con efectos sobre dinámicas ecológicas tales como la herbivoría, el ciclado de nutrientes, la erosión y la regulación de regímenes hídricos (Estes, et al., 2013, Dirzo, et al,2014, Ripple, et al., 2014).

La evaluación del riesgo de extinción de especies es fundamental para su conservación. El saber cuáles especies se encuentran en riesgo y las causas de amenaza permite orientar las acciones de conservación de manera apropiada y efectiva (Rodrigues, et al., 2006). Estas evaluaciones de riesgo, publicadas en documentos llamados listas o libros rojos, sirven de soporte a la definición de prioridades de conservación, la declaración de áreas protegidas, la formulación de políticas, el diseño de planes de conservación, la asignación de recursos económicos, la orientación de procesos de educación y la generación de conciencia pública sobre la vulnerabilidad de la naturaleza (UICN, 2003, Rodrigues, et al., 2006, Farrier, Whelan \& Mooney, 2007). La evaluación periódica del riesgo de extinción de las especies es indispensable para determinar el progreso o el retroceso en el estado de las especies y, por lo tanto, la efectividad de las políticas de conservación (UICN, 2003, Farrier, et al., 2007, Amaya-Espinel, et al., 2011).

Los libros rojos proveen una amplia y útil información obtenida mediante la recopilación del conocimiento en el cual se basan las evaluaciones (Rodrigues, et al., 2006). Su metodología se fundamenta en el sistema de categorías y criterios de la UICN, el cual es uno de los métodos más aceptados para evaluar el riesgo de extinción de las especies (Mace, et al., 2008). Los criterios utilizados cuantifican si está ocurriendo una rápida reducción del tamaño poblacional (criterio A), si el área de distribución de una especie es pequeña y está severamente fragmentada, o en disminución, o si fluctúa (criterio B); si la población es pequeña y está en disminución (criterio C); si la población o la distribución es muy pequeña y hay una amenaza plausible (criterio D), y, cuando se cuenta con suficiente información, si el análisis de viabilidad poblacional indica una gran probabilidad de desaparición (criterio E). Las categorías en las cuales se clasifica a las especies una vez evaluadas son las siguientes: extinta (EX), extinta en vida silvestre (EW), en peligro crítico
(CR), en peligro (EN), vulnerable (VU), casi amenazada (NT), datos insuficientes (DD) y preocupación menor (LC). Las especies en las categorías CR, EN y VU se consideran amenazadas. Por último, hay muchas especies que no han sido evaluadas (NE) (IUCN, 2001, 2012).

Colombia ha sido un país muy activo en la evaluación del riesgo de extinción de sus especies de flora y fauna. Entre el 2002 y el 2007, se publicaron 15 libros de especies amenazadas de plantas y animales terrestres, marinos y dulceacuícolas (Ardila, et al., 2002, Calderón, et al., 2002; Castaño-Mora, 2002, Linares \& Uribe-Meléndez, 2002, Mejía \& Acero, 2002, Mojica, et al., 2002, Renjifo, et al., 2002, Rueda-Almonacid, et al., 2004, Calderón, et al., 2005, García \& Galeano, 2006; Rodríguez-Mahecha, et al., 2006; Amat, et al., 2007; Calderón-Sáenz, 2007; Cárdenas \& Salinas, 2007; García, 2007). En la segunda década del siglo XXI se publicaron tres nuevos libros rojos: peces dulceacuícolas (Mojica, et al., 2012), aves (Renjifo, et al., 2014, 2016), y peces marinos (Chasqui, et al., 2017), en los cuales se volvió a evaluar el riesgo de las especies de esos grupos taxonómicos. La lógica de evaluación del riesgo de las especies se ha orientado recientemente a la evaluación del riesgo de extinción de ecosistemas (Rodríguez, et al., 2007, Keith, et al., 2013), y Colombia es uno de los primeros países en donde esto se está haciendo (Etter, et al., 2015).

Se han desarrollado diversas aproximaciones para monitorizar el estado de la biodiversidad global, especialmente después de la creación de la Convención sobre la Diversidad Biológica (Convention on Biological Diversity, CBD) en 1992. Por ejemplo, el indicador de "Planeta Vivo" (Living Planet Index, LPI), creado por el Fondo Mundial para la Naturaleza (Wild Wildlife Fund, WWF) en 1998, se utiliza para monitorizar las tendencias en la biodiversidad. Mide las tendencias poblacionales usando datos de series de tiempo para calcular tasas de cambio en las poblaciones de especies silvestres de vertebrados terrestres, marinos y dulceacuícolas (Loh \& Wackernagel, 2004, Loh, et al., 2005) y es considerado como uno de los conjuntos de datos más completos de observaciones sobre la abundancia de las poblaciones (Proença, et al., 2017). Al igual que los libros rojos, el LPI transmite de una forma sencilla al público no experto (como los responsables de decisiones, los políticos, el público general), la información sobre las tendencias de la biodiversidad (Collen, et al., 2009).

Las aves son un grupo muy diverso que incluye las 10.672 especies existentes (156 se han extinguido recientemente), pertenecientes a 40 órdenes y 238 familias (Gill \& Donsker, 2017). Además, las aves son de gran importancia evolutiva y ecológica (Şekercioğlu, 2006, Wenny, et al., 2011). La pérdida de las aves trae consigo un detrimento de los servicios ecosistémicos que proveen (como la polinización y la dispersión de semillas), con los cuales contribuyen a la regeneración de los ecosistemas; al control de poblaciones de invertebrados y microvertebrados plaga en sistemas productivos y en ecosistemas silvestres; al control de malezas 
en sistemas agrícolas; a la disposición de cadáveres, basuras y ciclado de nutrientes; a la relocalización de nutrientes, y a la provisión de cavidades de anidación para otras especies (Şekercioğlu, 2006, Whelan, et al., 2015). Las aves son también indicadoras de la salud de los ecosistemas (Carson, 1962, Gregory \& van Strien, 2010).

Colombia es coloquialmente conocido como el país de las aves porque es el de mayor riqueza de especies en el mundo (BirdLife International, 2017) y su tasa de descubrimiento de especies es de una nueva por año (Franco, et al., 2009). Las aves han sido el grupo con más evaluaciones de riesgo de extinción en el país. El primer inventario de especies que podrían encontrarse en riesgo fue la lista azul de Hilty (1985), seguida por cinco evaluaciones de riesgo (Rodríguez-Mahecha, et al., 1986, Renjifo, 1998, Negret, 2001, Renjifo, et al., 2002, Renjifo, et al., 2014, Renjifo, et al., 2016; los dos últimos corresponden a un mismo proceso de evaluación).

En este trabajo analizamos la evolución de las listas y libros rojos de aves en Colombia en cuanto a sistema de evaluación, métodos y composición. Así mismo, comparamos analíticamente los cambios en el estado de conservación de las aves entre el 2002 y el 2016. Para las especies amenazadas en el presente (Renjifo, et al., 2014, Renjifo, et al., 2016), analizamos la prevalencia de los tipos de amenazas directas, estimamos la proporción de especies amenazadas por cada familia y determinamos cuáles tienen mayor propensión a la extinción por selectividad taxonómica.

\section{Métodos}

Evolución en la evaluación de riesgo de extinción. Analizamos cada uno de los documentos publicados con evaluaciones de riesgo de las especies de aves en Colombia para determinar el sistema de evaluación y los métodos utilizados. Sistematizamos los listados de especies amenazadas de las cinco evaluaciones de riesgo: Rodríguez-Mahecha, et al., 1986, Renjifo, 1998, Negret, 2001, Renjifo, et al., 2002, Renjifo, et al., 2014 y Renjifo, et al., 2016. Como los dos volúmenes del libro rojo más reciente (2014 y 2016) hacen parte de un mismo ciclo de evaluación para las especies de aves del país, nos referiremos a esta evaluación como una sola (Renjifo, et al., 2016). Revisamos la taxonomía vigente siguiendo al Comité Suramericano de Clasificación (South American Classification Committee, SACC) de la Unión de Ornitólogos Americanos (versión 4, agosto 2016, Remsen, et al., 2016), para detectar cambios taxonómicos entre los distintos periodos de tiempo y unificar los listados según las sinonimias.

Cambios en la composición de la avifauna amenazada entre 2002 y 2016. Revisamos los cambios de las categorías entre los listados de Renjifo, et al., 2002 y Renjifo, et al., 2016, y contabilizamos los cambios que corresponden a una mejoría en el estado (downlist), empeoramiento (uplist) o sin cambios. Comparamos los cambios en las proporciones de especies amenazadas bajo los criterios A, B, C y D entre los años 2002 y 2016. Examinamos para cada periodo de tiempo cuántas especies resultaron amenazadas según cada criterio teniendo en cuenta todas las categorías de amenaza en las que se encuentra cada especie. Por ejemplo, el loro orejiamarillo, Ognorhynchus icterotis, se encuentra en peligro según el criterio $\mathrm{C}$ (C2a(i)) y es vulnerable según los criterios B y D (B2ab(v); D1), por lo tanto, se incluyó en el conjunto de especies amenazadas bajo los criterios B, C y D. Por último, determinamos la proporción de especies en 2002 y 2016 para las cuáles no había estimaciones del tamaño poblacional, y establecimos de cuáles había información que permitiera hacer dichas estimaciones después del 2002.

Proporción de especies amenazadas. Calculamos la proporción de especies amenazadas en el país y por familia de aves residentes permanente o estacionalmente en Colombia. Para esto añadimos al listado del SACC para Colombia las especies de San Andrés, según el listado de Pacheco-Garzón (2012), y las de Providencia, según la lista de chequeo de las aves del mundo Avibase (disponible en http://avibase.bsceoc.org/checklist.jsp?lang=ES\&region= copr\&list=ioc). Luego excluimos del listado las especies transeúntes, accidentales y aquellas de las cuales se sabe que su población nacional es de menos del $2 \%$ de la población global, dado que estos tipos de especies no deben ser evaluadas a escala nacional (UICN, 2003, 2012). Teniendo en cuenta que para las especies que se clasifican como DD, el riesgo de extinción es desconocido (podrían o no estar amenazadas), eliminamos la incertidumbre que introducen dichas especies, así: Proporción amenaza $=\frac{C R+E N+V U}{N-D D}$, donde $\mathrm{N}$ es el total de especies evaluadas. Este método se ha utilizado en diversos estudios sobre riesgo de extinción (Schipper, et al., 2008, Clausnitzer, et al., 2009, Hoffmann, et al., 2010, Böhm, et al., 2013). Siguiendo a Böhm, et al. (2013), calculamos el rango superior de esta proporción asumiendo que las especies DD estaban amenazadas (Proporción $\left.=\frac{C R+E N+V U+D D}{N}\right)$, y el rango inferior asumiendo que las especies DD no lo estaban (Proporción ${ }_{\text {amenaza }}=\frac{C R+E N+V U}{N}$ ).

Con el fin de determinar si las especies amenazadas estan aleatoriamente distribuidas entre las familias o no, es decir, si hay selectividad taxonómica en el riesgo de extinción, hicimos una prueba exacta de Fisher (Sokal \& Rohlf, 1995) utilizando el programa R (R Core Team 2011) para las familias de aves con cinco o más especies en Colombia $(n=50)$. Comparamos el número observado de especies amenazadas en cada familia con una distribución nula de frecuencias de 10.000 aleatorizaciones (valor esperado de proporción de especies amenazadas de 0,08 y de especies no amenazadas de 0,92). El análisis se hizo con un intervalo de confianza de $95 \%$. Las familias con valores de $\mathrm{p}<0,05$ se consideraron por encima del valor esperado de amenaza.

Amenazas. Para analizar la prevalencia de los tipos de amenazas directas en el presente, examinamos la información de la sección "Amenazas" de las fichas de síntesis de la información y de la sección "Situación de la especie" en 
Renjifo, et al., (2014, 2016), y utilizamos la clasificación de amenazas directas (IUCN-CMP, versión 1.1, Salafsky, et al., 2008) para cada una de ellas, así: 1) desarrollo residencial y comercial; 2) agricultura y acuacultura; 3 ) producción de energía y minería; 4) transporte y corredores de servicio; 5) uso de recursos biológicos; 6) intrusión y disturbios por humanos; 7) modificaciones de sistemas naturales; 8) especies invasoras y otras especies problemáticas; 9) contaminación; 10) eventos geológicos, y 11) cambio climático y clima severo. En la categoría de “Agricultura” añadimos los cultivos ilícitos como subcategoría, pues son una amenaza de gran relevancia en Colombia. Una especie suele estar amenazada por diversas amenazas directas, dado que éstas no son mutuamente excluyentes.

\section{Resultados}

Sistemas de evaluación y métodos. En cada una de las cinco evaluaciones (Rodríguez-Mahecha, et al., 1986, Renjifo, 1998, Negret, 2001, Renjifo, et al., 2002, y Renjifo, et al., 2016), se utilizó de manera implícita o explícita el sistema de la UICN vigente en su momento. Tanto RodríguezMahecha, et al. (1986) como Negret (2001) utilizaron un sistema de categorías, pero no de criterios, pues estos fueron desarrollados posteriormente. Rodríguez-Mahecha, et al. (1986) elaboraron la lista de especies y subespecies con base en el criterio experto sobre la percepción del grado de amenaza. No obstante, el criterio de inclusión de subespecies utilizado por estos autores no es explícito. Las categorías de este listado son "Extinta” y "En Vías de Extinción" (Tabla 1S, https://www.raccefyn.co/index.php/ raccefyn/article/downloadSuppFile/461/2388). La obra de Álvaro José Negret se escribió en los años ochenta y noventa y fue publicada póstumamente (Negret, 2001). En ella no se encuentra una descripción de métodos, lo cual indica que se hizo con base en el criterio experto. Las especies se categorizaron como "Extintas" (E-1), “Amenazadas” (E-2), "Vulnerables" (V-3), "Raras" (R-4) e "Indeterminadas" (I5) (Tabla 2S, https://www.raccefyn.co/index.php/raccefyn/ article/downloadSuppFile/461/2389).

A partir de Renjifo (1998) se utilizó el sistema de categorías y criterios desarrollado y refinado progresivamente por la UICN entre 1991 y 2001 (Mace \& Lande, 1991, UICN 2001). Todas estas evaluaciones se hicieron a nivel de especie. En Renjifo (1998) las evaluaciones partieron de un listado de especies potencialmente amenazadas recopilado de fuentes publicadas e inéditas. Las evaluaciones se hicieron con base en el criterio experto a partir de la compilación de información sobre la distribución, la sensibilidad y la situación de cada especie a nivel global y nacional, y utilizando como referente la situación de los hábitats de las especies y la distribución de los ecosistemas terrestres del país (Etter, 1993). Tanto la recopilación de la información como la evaluación del riesgo fueron responsabilidad de un solo autor. A partir de Renjifo, et al. (2002) el compendio de información de las especies potencialmente amenazadas fue ampliamente participativo, pues se convocaron investigadores nacionales e internacionales para elaborar las fichas de síntesis de la información sobre las especies con datos de la distribución, la ecología, las amenazas y las medidas de conservación tomadas. Además, se invitó a investigadores, conservacionistas y observadores de aves a aportar registros de distribución de las especies. Esta investigación estuvo a cargo de un equipo de cinco investigadores y se sirvió de las síntesis de información escritas por 47 colaboradores y los aportes de registros de información de 118 personas. En el estudio de Renjifo, et al. (2002) se hicieron por primera vez, en el país y en el mundo, estimaciones cuantitativas de las áreas de distribución y los tamaños poblacionales de las especies utilizando sistemas de información geográfica. Para estimar la distribución de las especies se usaron modelos simples de superposición de capas de modelos digitales de elevación, modelos de ecosistemas originales (Etter, et al., sin publicar, versión 1999), de ecosistemas actuales (Etter, 1998, Fundación Pro-Sierra Nevada de Santa Marta, 1998), y la distribución conocida e inferida de las especies. El tamaño de las poblaciones se estimó utilizando estimaciones del área de hábitats remanentes por especie y densidades poblacionales conocidas. Las estimaciones de la velocidad de pérdida de hábitat y el cambio poblacional se basaron en el criterio experto.

La evaluación más reciente (Renjifo, et al., 2014, Renjifo, et al., 2016) estuvo a cargo de un equipo de siete investigadores, y se apoyó en las síntesis de información escritas por 155 colaboradores y los aportes de registros de información de 142 personas. Para esta evaluación se hicieron estimaciones cuantitativas de distribución de las especies basadas en modelos de distribución establecidos mediante el uso de variables climáticas y registros biológicos. Los modelos se refinaron mediante la consideración de la cobertura vegetal y el criterio experto. Para la obtención de capas de variables climáticas necesarias para los modelos de distribución, se interpolaron datos provenientes de la red de estaciones del IDEAM a lo largo de cuatro décadas. Con los modelos de distribución se estimó el tamaño del área de distribución de cada especie. Con la información multitemporal sobre la cobertura vegetal con relación a la distribución de cada especie, se estimaron la magnitud y la velocidad de cambio en la extensión de los hábitats y las poblaciones de las especies. Se calculó el tamaño poblacional de las especies con base en estimaciones del área de ocupación de cada una de ellas y las densidades poblacionales reportadas en la literatura. Cuando una especie es endémica su evaluación corresponde a una evaluación global, pero cuando no lo es, se requiere un ajuste de carácter regional porque la probabilidad de extinción puede no ser independiente de lo que ocurra en países vecinos; para esto se utilizaron los métodos publicados por la UICN (2003). Este ajuste no se aplicó a las evaluaciones en el estudio de Renjifo, et al. (2002), dado que los métodos no estaban disponibles en ese momento. Las subespecies se 
incluyeron en el análisis si había evidencia que indicara que ese taxón podría ser tratado como especie válida que debería ser evaluada por precaución (ver Laverde, 2014 en Renjifo, et al., 2014).

Cambios en la composición de las listas. El número de especies incluidas en las listas rojas ha aumentado a través del tiempo (Tabla 1). Mientras en la primera evaluación de riesgo de extinción Rodríguez-Mahecha, et al. (1986) consideraron que 62 especies estaban "En Vías de Extinción” y que dos taxones estaban extintos (la especie Podiceps andinus y la subespecie Anas georgica nicefori) (Tabla 1S), en la evaluación de Negret (2001), 89 especies se consideraron en las categorías "Amenazada" y "Vulnerable" y tres taxones se consideraron "Extintos" (P. andinus, Anas georgica niceforoi y Sporophila insulata) (Tabla 2S). Más adelante, en la primera evaluación de Renjifo (1998), se concluyó que 83 especies estaban amenazadas (CR, EN y VU). Este número aumentó a 112 (Renjifo, 2002) y actualmente es de 140 (años 2014 y 2016) (Tablas 1 y 2).

Cambios entre el 2002 y el 2016. De las 112 especies en alguna categoría de amenaza en el año 2002, 54 (48 \%) permanecen en la misma categoría, $16(14 \%)$ subieron a una categoría (empeoraron) de mayor riesgo y 36 (33\%) bajaron de categoría (mejoraron) (Tabla 2). De las especies que bajaron de categoría, 11 (10\%) pasaron de estar en una categoría de amenaza a NT o LC. Una especie pasó de CR a DD (Cypseloides lemosi) y cinco no han sido evaluadas recientemente (Renjifo, et al., 2014, Renjifo, et al., 2016), tres de ellas debido a cambios en la taxonomía (los casos de sinonimización mencionados más adelante) y dos, porque se siguió el criterio de filtración de la UICN que indica que no se deben evaluar especies cuya población en el país sea menor de $2 \%$ de la población global (IUCN, 2012).

Hasta este punto la tendencia de los cambios entre 2002 y 2016 podría parecer positiva. No obstante, 27 especies entraron por primera vez a alguna categoría de amenaza (CR, EN, VU) y siete pasaron de estar en la categoría de preocupación menor a casi amenazadas (Tabla 2). En conjunto, todos estos cambios muestran una tendencia claramente negativa. Los cambios de categoría de una especie pueden deberse a una mayor disponibilidad de información, a cambios en los métodos analíticos o a cambios genuinos en su estado de conservación. El análisis detallado para determinar el tipo de cambio que ha tenido cada una de las especies va más allá del objetivo de este trabajo y será publicado como un indicador de listas rojas de aves para Colombia. (Sobre los indicadores para la elaboración de listas rojas, véase a Butchart, et al.; 2007).

Además, encontramos que de las especies descritas como nuevas para la ciencia después del 2002, ocho se encuentran amenazadas. Estas especies son Henicorhina negreti (Salaman, et al., 2003), Scytalopus rodriguezi (Krabbe, et al., 2005a), Scytalopus stilesi (Cuervo, et al., 2005), Eriocnemis isabellae (Cortés-Diago, et al., 2007), Atlapetes blancae (Donegan, 2007), Grallaria urraoensis
Tabla 1. Número y porcentaje de especies en cada categoría en los años 1986, 1998, 2002 y 2016.

\begin{tabular}{lcccc}
\hline \multirow{2}{*}{ Categoría } & $\mathbf{1 9 8 6}$ & $\mathbf{1 9 9 8}$ & $\mathbf{2 0 0 2}$ & $\mathbf{2 0 1 6}$ \\
\cline { 2 - 5 } & N/A & $13(15,7)$ & $19(17,0)$ & $17(12,1)$ \\
\hline CR & N/A & $24(28,9)$ & $44(39,3)$ & $56(40,0)$ \\
\hline EN & N/A & $46(55,4)$ & $49(43,8)$ & $67(47,9)$ \\
VU & $\mathbf{6 2 ~ ( 1 0 0 )}$ & $\mathbf{8 3 ( 1 0 0 )}$ & $\mathbf{1 1 2 ( 1 0 0 )}$ & $\mathbf{1 4 0}(\mathbf{1 0 0})$ \\
Total amenazadas & 2 & 1 & 1 & 1 \\
EX & N/A & 75 & 40 & 28 \\
NT & N/A & 5 & 9 & 9 \\
DD & 2 & 81 & 50 & 38 \\
Total EX+NT+DD & $\mathbf{6 4}$ & $\mathbf{1 6 4}$ & $\mathbf{1 6 2}$ & $\mathbf{1 7 8}$ \\
\hline Total especies & & &
\end{tabular}

CR: en peligro crítico; EN: en peligro; VU: vulnerable; N/A: no aplica; EX: extinta, NT: casi amenazada; DD: datos insuficientes

(Carantón \& Certuche, 2010), Thryophilus sernai (Lara, et al., 2012) y Scytalopus perijanus (Avendaño, et al., 2015).

Cambios taxonómicos. Los cambios en la taxonomía dificultan la comparación de los análisis de riesgo de extinción de algunos taxones a través del tiempo. Por ejemplo, la sinonimización, que ocurre cuando taxones antes considerados especies válidas son posteriormente considerados subespecies. Este es el caso de Crypturellus columbianus y C. saltuarius, antes tratados como especies plenas, pero que hoy se consideran subespecies de C. erythropus (Remsen, et al., 2016). Tal cambio taxonómico implica que el ámbito de distribución de C. erythropus es más amplio y se considera en bajo riesgo. El caso contrario lo representan las divisiones taxonómicas. Siete taxones (Anthocephala berlepschi, Clibanornis rufipectus, Oxypogon stubelii, O. cyanolaemus, Scytalopus sanctaemartae, S. canus y Troglodytes monticola), fueron tratados como subespecies en el año 2002 y, posteriormente, elevados a nivel de especie. Al evaluarlas, calificaron como amenazadas (para más detalles ver el Apéndice 4 de Renjifo, et al., 2016).

Otro tipo de cambio se debe a la aclaración del estatus de algunas aves. Sporophila insulata se consideró como una especie válida, dada por extinta en la evaluación de Negret (2001), y como amenazada en la de Renjifo (1998) y Renjifo, et al. (2002), pero hoy se piensa que es un híbrido entre $S$. telasco y S. minuta (De las Casas, et al., 2004) o un morfo de color de S. telasco (Stiles, 2004). Lo opuesto ocurrió con Coeligena orina, cuyo estatus permaneció incierto por décadas. El hallazgo de poblaciones en la cordillera Occidental en el año 2004 permitió la confirmación de su validez como especie (Krabbe, et al., 2005b).

La inestabilidad taxonómica ocasionada por el avance en el conocimiento, la complejidad de la clasificación de los organismos y el desacuerdo entre las autoridades taxonómicas en cuanto a los criterios de aceptación de las especies como válidas, puede interferir con una comunicación efectiva, así como con los programas de conservación, los 
Tabla 2. Especies y subespecies de aves de Colombia listadas como amenazadas o casi amenazadas en tres periodos de tiempo. No aplica (N/A) corresponden a taxones no válidos en el pasado o en el presente, nuevos registros de especies para Colombia o para la ciencia en un tiempo posterior a las evaluaciones en cada columna. $\mathrm{NE}=$ No evaluada. Las especies endémicas están marcadas con un asterisco. La taxonomía y los nombres científicos siguen SACC (2016).

\begin{tabular}{|c|c|c|c|c|}
\hline Familia & Taxón & Renjifo 1998 & Renjifo et al. 2002 & Renjifo et al. 2016 \\
\hline \multirow[t]{7}{*}{ Tinamidae } & Nothocercus bonapartei & VU C2a & LC & LC \\
\hline & Tinamus tao & VU C2a & LC & LC \\
\hline & Tinamus osgoodi & $\mathrm{CR} \mathrm{B} 1+2 \mathrm{c} ; \mathrm{C} 2 \mathrm{a}$ & EN B2ab(iii,v); C2a(i) & EN B1ab(ii,iii,v)+2ab(ii,iii,v); C2a(i) \\
\hline & $\begin{array}{l}\text { Crypturellus obsoletus } \\
\text { castaneus*1 }\end{array}$ & N/A & N/A & CR C2a(i) \\
\hline & $\begin{array}{l}\text { Crypturellus erythropus } \\
\text { columbianus }^{2}\end{array}$ & NT & EN A4cd & N/A \\
\hline & $\begin{array}{l}\text { Crypturellus erythrops } \\
\text { saltuarius }^{2}\end{array}$ & $\mathrm{CR} \mathrm{B} 1+2 \mathrm{c} ; \mathrm{C} 2 \mathrm{~b}$ & CR B2ab(i,ii,iii,iv,v); D1 & N/A \\
\hline & Crypturellus kerriae & $\mathrm{VU} B 1+2 \mathrm{c} ; \mathrm{C} 2 \mathrm{a}$ & VU B1ab(i,ii,iv) & VU C2a(i) \\
\hline Anhimidae & Chauna chavaria & NT & VU C2a(i) & VU C2a(i) \\
\hline Procellariidae & Pterodroma phaeopygia ${ }^{3}$ & $\mathrm{CR} A 1 \mathrm{ac}+2 \mathrm{c}$ & CR A2ace & NE \\
\hline \multirow[t]{7}{*}{ Anatidae } & Oressochen jubatus & NT & NT A2, cd $+3 \mathrm{~cd}$ & VU A2cd+4cd; C2a(ii) \\
\hline & Sarkidiornis melanotos & LC & EN B2ab(ii,iii) & EN B2ab(iii,v); C2a(i) \\
\hline & Anas georgica & LC & EN B2ab(ii,iii,v); C1 & VU A2cd+4cd; B2ab(iii,v); C2a(ii) \\
\hline & Anas bahamensis & $\mathrm{LC}$ & $\mathrm{LC}$ & NT A2cd+4cd; B2ab(iii,v); C2a(ii) \\
\hline & Anas cyanoptera & VU A1a; C2a & EN B2ab(ii,iii,iv); C1 & EN B2ab(iii,v); C2a(i) \\
\hline & Netta erythrophthalma & CR A1a; C1 & CR C2a(i) & CR D \\
\hline & Oxyura jamaicensis & $\mathrm{LC}$ & EN B2ab(ii,iii); C1+2a(i) & EN B2ab(iii,v); C2a(ii) \\
\hline \multirow[t]{9}{*}{ Cracidae } & Penelope ortoni & VU A1cd $+2 c d$ & $\mathrm{VU} C 1+2 \mathrm{a}(\mathrm{i})$ & VU C2a(i) \\
\hline & Penelope perspicax* & EN C2a & EN B2ab(ii,iii,v); C2a(i) & EN C2a(i) \\
\hline & Aburria aburri & NT & NT & LC \\
\hline & Ortalis erythroptera & LC & VU A1cd+2cd; B1a+2ab(i,ii,iii,iv); C1 & NT B1ab(iii,v)+2ab(iii,v) \\
\hline & Crax rubra & LC & LC & VU A2cd $+4 c d$ \\
\hline & Crax alberti* & CR C2a & CR A4cd & CR A2cd $+4 c d$ \\
\hline & Crax daubentoni & LC & VU C1+2a(i) & EN A2cd+4cd; C2a(i) \\
\hline & Crax globulosa & VU A1cd+2cd & CR B2a(i,ii,iii,iv,v); C2a(ii); D1 & EN B1ab(iii,iv)+2ab(iii,iv); C2a(i) \\
\hline & Pauxi pauxi & $\mathrm{EN} \mathrm{B} 1+2 \mathrm{c}$ & VU A2cd+4cd; B2ab(ii,iii); C2a(i) & EN A2cd $+4 c d$ \\
\hline \multirow[t]{5}{*}{ Odontophoridae } & Odontophorus atrifrons & NT & VU B2ab(iii) & NT B2ab(ii,iii,v) \\
\hline & Odontophorus hyperythrus & NT & NT B1+2abcde; C2a & LC \\
\hline & Odontophorus melanonotus & NT & VU A4c; B1ab(i,iii)+2ab(i,iii) & EN B1ab(ii,iii,v) \\
\hline & Odontophorus dialeucos & VU D2 & $\begin{array}{l}\text { EN B1ab(i,ii,iii,iv,v)+2ab(i,ii,iii,iv,v); } \\
\text { C2a(ii) }\end{array}$ & EN B1ab(iii,v)+2ab(iii,v); C2a(ii) \\
\hline & Odontophorus strophium* & $\mathrm{EN} \mathrm{B} 1+2 \mathrm{c} ; \mathrm{C} 2 \mathrm{a}$ & CR B2ab(ii,iii,iv); C2a(ii) & EN B2ab(i,ii,iii,iv,v) \\
\hline \multirow[t]{2}{*}{ Podicipedidae } & Podiceps andinus* & EX & $\mathrm{EX}$ & EX \\
\hline & Podiceps occipitalis & VU C2a & EN B2ab(ii,iii,iv) & CR B2ab(iii,v); C2a(i) \\
\hline Phoenicopteridae & Phoenicopterus ruber & LC & VU B1ab(i,iii); C2a(ii) & EN A2cd; B2ab(iii,v); C2a(ii) \\
\hline \multirow[t]{2}{*}{ Sulidae } & Sula granti ${ }^{4}$ & N/A & VU D2 & VU D2 \\
\hline & Sula leucogaster & LC & LC & EN B2ab(i,ii,iii,iv,v); C2a(i) \\
\hline \multirow[t]{4}{*}{ Ardeidae } & Egretta rufescens & LC & LC & VU C2a(ii) \\
\hline & Tigrisoma fasciatum & NT & LC & LC \\
\hline & Agamia agami & NT & LC & LC \\
\hline & Zebrilus undulatus & NT & LC & LC \\
\hline Cathartidae & Vultur gryphus & EN A1a; C2a & EN D1 & CR C2a(i) \\
\hline
\end{tabular}




\begin{tabular}{|c|c|c|c|c|}
\hline \multirow[t]{10}{*}{ Accipitridae } & Morphnus guianensis & NT & NT A $2 c d+3 c d$ & NT A2cd+4cd; C2a(ii) \\
\hline & Harpia harpyja & NT & NT A2cd+3cd & NT A2cd+4cd; C2a(ii) \\
\hline & Spizaetus isidori & NT & EN C2a & EN A2cd $+4 c d ; C 1$ \\
\hline & Circus cinereus & LC & LC & EN B2ab(i,ii,iii,v); C2a(ii); D \\
\hline & Accipiter collaris & NT & $\mathrm{NT} \mathrm{C1+2a(i)}$ & NT C2a(i) \\
\hline & Accipiter poliogaster & NT & LC & LC \\
\hline & Cryptoleucopteryx plumbea & NT & NT B1+2abcde; $\mathrm{C} 1+2 \mathrm{a}$ & NT C2a(ii) \\
\hline & Leucopternis semiplumbeus & NT & LC & $\mathrm{LC}$ \\
\hline & Buteogallus solitarius & NT & EN C2a(i) & CR C2a(i) \\
\hline & Spizastur melanoleucus & NT & LC & LC \\
\hline \multirow[t]{6}{*}{ Rallidae } & Coturnicops notatus & DD & DD & $\mathrm{DD}$ \\
\hline & Micropygia schomburgkii & NT & LC & LC \\
\hline & Rallus semiplumbeus* & $\mathrm{EN} \mathrm{B} 1+2 \mathrm{c} ; \mathrm{C} 2 \mathrm{a}$ & EN B2ab(ii,iii); C1+2a(i) & EN B2ab(ii,iii,v) \\
\hline & Aramides wolfi & VU Alc & VU A4c & LC \\
\hline & Mustelirallus colombianus & NT & DD & $\mathrm{DD}$ \\
\hline & Porphyriops melanops & VU A1a+2b; C2a & CR A2abce; B2ab(i,ii,iii,iv,v) & EN B2ab(iii,v); C2a(i) \\
\hline Scolopacidae & Gallinago imperialis & DD & $\mathrm{DD}$ & $\mathrm{DD}$ \\
\hline Laridae & Creagrus furcatus & LC & EN D1 & EN D \\
\hline \multirow[t]{4}{*}{ Columbidae } & Patagioenas leucocephala & LC & LC & NT B2ab(iii) \\
\hline & Leptotila conoveri* & EN C2a & EN C2a & VU B2ab(ii,iii,v); C2a(i) \\
\hline & Leptotila jamaicensis & $\mathrm{NE}$ & NE & CR B1ab(iii,v) \\
\hline & Zentrygon goldmani & NT & NT B1+2abcde & $\begin{array}{l}\text { VU B1ab(iii, v)+2ab(iii, v); C2a(i) } \\
\text {; D1 }\end{array}$ \\
\hline Cuculidae & Neomorphus radiolosus & EN C2a & VU C2a(i) & EN C2a(i) \\
\hline \multirow[t]{3}{*}{ Strigidae } & Glaucidium nubicola & LC & $\mathrm{VU} A 2 \mathrm{c}+3 \mathrm{c}$ & VU B2ab(ii,iii,v); C2a(i) \\
\hline & Aegolius harrisii & NT & LC & LC \\
\hline & Megascops colombianus & NT & NT B1ab(iii) + 2ab(iii) & LC \\
\hline Caprimulgidae & Nyctiphrynus rosenbergi & NT & NT A2c $+3 c$ & LC \\
\hline \multirow[t]{3}{*}{ Apodidae } & Cypseloides cherriei & $\mathrm{NE}$ & $\mathrm{DD}$ & $\mathrm{DD}$ \\
\hline & Cypseloides lemosi & $\mathrm{VU} C 2 \mathrm{~b}$ & CR B2ab(iii,iv); C1 & $\mathrm{DD}$ \\
\hline & Tachornis furcata & $\mathrm{NE}$ & DD & DD \\
\hline \multirow[t]{18}{*}{ Trochilidae } & Heliangelus zusii* & CR D1 & DD & CR-PE C2a(i) \\
\hline & Lophornis stictolophus & NT & LC & $\mathrm{LC}$ \\
\hline & Phlogophilus hemileucurus & NT & NT & NT B1ab(ii,iii,v)+2ab(ii,iii,v) \\
\hline & Anthocephala floriceps* & NT & VU B2ab(iii) & VU B1ab(ii,iii,v)+2ab(ii,iii,v) \\
\hline & Anthocephala berlepschi*5 & N/A & N/A & VU B2ab(ii,iii,v) \\
\hline & Oxypogon stubelii* 6 & N/A & N/A & EN B1ab(ii,iii,v)+2ab(ii,iii,v) \\
\hline & Oxypogon cyanolaemus* 6 & N/A & N/A & EN B1ab(ii,iii,v)+2ab(ii,iii,v) \\
\hline & Metallura iracunda & NT & EN B1ab(iii)+2ab(iii) & NT B1ab(ii,iii,v)+2ab(ii,iii,v); C2a(ii) \\
\hline & Haplophaedia lugens & NT & NT B1ab(iii)+2ab(iii) & NT B1ab(ii,iii,v)+2ab(ii,iii,v) \\
\hline & Eriocnemis isabellae* 7 & N/A & N/A & CR B1ab(ii,iii,v)+2ab(ii,iii,v) \\
\hline & Eriocnemis derbyi & NT & NT A2c+3c; B1+2abcde & LC \\
\hline & Eriocnemis godini & $\mathrm{CR}$ B1+2c; C2b; D1 & $\mathrm{DD}$ & CR-PE C2a(i) \\
\hline & Eriocnemis cupreoventris & LC & NT B1+2abce & LC \\
\hline & Eriocnemis mirabilis* & VU D1+2 & CR B1ab(i,ii,iv)+2ab(i,ii,iv) & EN B1ab(iii,v)+2ab(iii,v) \\
\hline & Coeligena prunellei* & $\mathrm{VU} A 1 \mathrm{c}+2 \mathrm{c} ; \mathrm{C} 1+2 \mathrm{a}$ & EN B2ab(iii,iv) & NT B2ab(ii,v) \\
\hline & Coeligena orina*8 & N/A & N/A & EN B1ab(iii,v)+2ab(iii,v) \\
\hline & Heliodoxa gularis & NT & NT A2c+3c; B1+2abce & NT B1ab(ii,iii,iv,v)+2ab(ii,iii,iv,v) \\
\hline & Acestrura bombus ${ }^{9}$ & N/A & $\mathrm{VU} C 1+2 \mathrm{a}$ & $\mathrm{NE}$ \\
\hline
\end{tabular}




\begin{tabular}{|c|c|c|c|c|}
\hline & $\begin{array}{l}\text { Campylopterus } \\
\text { phainopeplus* }\end{array}$ & NT & EN B1ab(i,iii) & VU Blab(iii,v) \\
\hline & Amazilia castaneiventris* & EN A1c & CR B2a(iii,iv) & EN B2ab(iii,v) \\
\hline & Goethalsia bella & NT & EN B1ab(ii,iii,v)+2ab(ii,iii,v) & VU B1ab(iii)+2ab(iii) \\
\hline & Lepidopyga lilliae* & CR C2b; D1 & CR C2a(i) & EN B2ab(ii,iii,v) \\
\hline Galbulidae & Galbula pastazae & VU C2a & VU B2ab(ii,iii) & VU C2a(ii) \\
\hline \multirow[t]{2}{*}{ Bucconidae } & Bucco noanamae* & NT & NT B1+2abce & NT A2c+4c; C2a(ii) \\
\hline & Micromonacha lanceolata & NT & LC & LC \\
\hline \multirow[t]{3}{*}{ Capitonidae } & Capito squamatus & NT & NT A4c; B1ab(ii,iii,iv,v) & VU A4ce; B1ab(ii,iii,v) \\
\hline & Capito hypoleucus* & EN C2a & EN A4c & EN A4ce \\
\hline & Capito quinticolor & VU A1c & NT A4c & NT A4c \\
\hline Semnornithidae & Semnornis ramphastinus & NT & NT B2ab(iii) & NT B2ab(ii,iii,iv,v) \\
\hline \multirow[t]{3}{*}{ Ramphastidae } & Andigena hypoglauca & NT & VU B2ab(ii,iii,v) & $\mathrm{VU} A 2 \mathrm{c}+4 \mathrm{c}$ \\
\hline & Andigena laminirostris & NT & $\begin{array}{l}\text { VU A2c+3c; B1ab(ii,iii,iv,v)+2ab(i,i } \\
\text { i,iii,iv,v) }\end{array}$ & EN Blab(ii,iii,v) \\
\hline & Andigena nigrirostris & NT & NT A3c & NT A2c $+4 c$ \\
\hline \multirow[t]{4}{*}{ Picidae } & Melanerpes pulcher & VU C2a & VU A4c & $\mathrm{LC}$ \\
\hline & Veniliornis callonotus & $\mathrm{NE}$ & $\mathrm{NE}$ & EN B1ab(ii,iii,v)+2ab(ii,iii,v); C2a(i); \\
\hline & Veniliornis chocoensis & NT & NT A2c+3c; B1+2abcde & LC \\
\hline & Campephilus gayaquilensis & $\mathrm{LC}$ & NT & EN B2ab(ii,iii,v); C2a(i,ii) \\
\hline \multirow[t]{2}{*}{ Falconidae } & Micrastur plumbeus & EN C2a & $\mathrm{NT} \mathrm{C} 1+2 \mathrm{a}$ & EN C1 \\
\hline & Falco deiroleucus & NT & $\mathrm{DD}$ & $\mathrm{DD}$ \\
\hline \multirow[t]{12}{*}{ Psittacidae } & Touit stictopterus & VU C2a & EN A4c; B2ab(iii,v) & VU C2a(i) \\
\hline & $\begin{array}{l}\text { Bolborhynchus } \\
\text { ferrugineifrons* }\end{array}$ & EN C2a & VU B1ab(iii)+2ab(iii); C2a(i) & VU B1ab(iii)+2ab(iii) \\
\hline & Hapalopsittaca amazonina & EN C1 & VU C2a(i) & VU C2a(i) \\
\hline & Hapalopsittaca fuertesi* & $\mathrm{CR} D 1+2$ & CR C2a(ii) & CR C2a(i) \\
\hline & Pyrilia pyrilia & VU A1a, C2a & VU A4c; C1 & NT C2a(ii) \\
\hline & Pionus fuscus & $\mathrm{NE}$ & $\mathrm{NE}$ & EN B1ab(iii)+2ab(iii) \\
\hline & Pyrrhura viridicata* & $\mathrm{VU} C 1+2 \mathrm{~b}$ & EN B2ab(iii); C2a(ii) & EN C2a(ii) \\
\hline & Pyrrhura calliptera* & $\mathrm{VU} A 1 \mathrm{c}+2 \mathrm{c} ; \mathrm{C} 1+2 \mathrm{a}$ & VU A4c & $\begin{array}{l}\text { VU A2cd+3cd+4cd; B2ab(i,ii,iii,iv,v); } \\
\text { C2a(ii) }\end{array}$ \\
\hline & Ara militaris & VU A1bc; C2a & VU A2cd $+3 c d$ & VU A2cd+4cd; C2a(i) \\
\hline & Ara ambiguus & VU A1bc; C2a & VU A2cd $+3 c d$ & EN A2cd+4cd; C1+2a(ii) \\
\hline & Leptosittaca branickii & $\mathrm{VU} A 1 \mathrm{c}+2 \mathrm{c} ; \mathrm{C} 1+2 \mathrm{a}$ & VU A2cd+4cd; C2a(i) & VU C2a(ii) \\
\hline & Ognorhynchus icterotis & CR C2a & CR B2ab(ii,iv,v); C1+2a(i) & EN C2a(i) \\
\hline \multirow[t]{3}{*}{ Thamnophilidae } & Clytoctantes alixii & $\mathrm{EN} \mathrm{C1}+2 \mathrm{a}$ & EN A4c; B2ab(ii,iv); C2a(i) & VU B2ab(iii) \\
\hline & Dysithamnus occidentalis & $\mathrm{VUC} 1+2 \mathrm{a}$ & VU B1ab(i,ii,iii,v) & VU C2a(i) \\
\hline & Xenornis setifrons & VU C2a & VU B2ab(ii,iii,v) & VU B2ab(iii) \\
\hline Conopophagidae & Pittasoma rufopileatum & LC & NT & LC \\
\hline \multirow[t]{9}{*}{ Grallariidae } & Grallaria gigantea & $\mathrm{VUA} 1 \mathrm{c}+2 \mathrm{c} ; \mathrm{C} 1+2 \mathrm{a}$ & EN B2ab(ii,iii,iv) & VU B2ab(ii,iii,v) \\
\hline & Grallaria alleni & $\mathrm{EN} \mathrm{B} 1+2 \mathrm{c} ; \mathrm{C} 2 \mathrm{a}$ & EN B2ab(ii,iii,iv) & EN B2ab(ii,iii,iv); C2a(i) \\
\hline & Grallaria bangsi* & NT & VU B1ab(i,ii,iii)+2ab(i,ii,iii) & VU B1ab(iii,v)+2ab(iii,v) \\
\hline & Grallaria kaestneri* & VU D2 & EN B1ab(i,ii,iii,iv)+2ab(i,ii,iii,iv) & $\begin{array}{l}\text { EN B1ab(ii,iii,iv,v)+2ab(ii,iii,iv,v); } \\
\text { C2a(i) }\end{array}$ \\
\hline & Grallaria rufocinerea & VU C2a & VU A4c & VU B2ab(ii,iii,v) \\
\hline & Grallaria urraoensis*10 & N/A & N/A & EN B1a(iii)+2a(iii); C2a(ii) \\
\hline & Grallaria milleri* & $\mathrm{EN} \mathrm{B} 1+2 \mathrm{c}$ & EN B2ab(ii,iii,iv,v) & EN B2ab(ii,iii) \\
\hline & Grallaricula cucullata & $\mathrm{VU} A 1 \mathrm{c}+2 \mathrm{c} ; \mathrm{C} 1+2 \mathrm{a}$ & NT & LC \\
\hline & Grallaricula lineifrons & NT & VU B1ab(ii,iii,v)+2ab(ii,iii,v) & LC \\
\hline
\end{tabular}




\begin{tabular}{|c|c|c|c|c|}
\hline \multirow[t]{6}{*}{ Rhinocryptidae } & Scytalopus sanctaemartae $* 11$ & N/A & N/A & VU B1ab(iii,v)+2ab(iii,v) \\
\hline & Scytalopus panamensis & VU D2 & EN B1ab(ii,iii,v)+2ab(ii,iii,v) & VU C2a(ii) \\
\hline & Scytalopus rodriguezi*12 & N/A & N/A & VU B1ab(ii,iii,v) \\
\hline & Scytalopus stilesi*13 & N/A & N/A & EN B2ab(ii,iii,v) \\
\hline & Scytalopus perijanus ${ }^{14}$ & N/A & N/A & VU B1ab(ii,iii,v)+2ab(ii,iii,v); C2a(i) \\
\hline & Scytalopus canus* 15 & N/A & N/A & EN B1ab(iii)+2ab(iii) \\
\hline \multirow[t]{8}{*}{ Furnariidae } & Drymotoxeres pucheranii & NT & NT A4c & $\mathrm{LC}$ \\
\hline & Clibanornis rufipectus $* 16$ & N/A & N/A & $\begin{array}{l}\text { VU B1ab(ii,iii,v)+2ab(ii,iii,v); } \\
\text { C1+2a(i) }\end{array}$ \\
\hline & Margarornis stellatus & $\mathrm{LC}$ & NT A2c $+3 c$ & LC \\
\hline & Margarornis bellulus & VU D2 & EN B1ab(ii,iii,v)+2ab(ii,iii,v); C2a(i) & VU B1ab(iii,v)+2ab(iii,v); C2a(ii) \\
\hline & Asthenes perijana & NT & EN B1ab(i,ii,iii)+2(i,ii,iii) & EN B1ab(iii,v)+2ab(iii,v); C2a(ii); D \\
\hline & Siptornis striaticollis & LC & NT A4c & LC \\
\hline & Synallaxis fuscorufa* & NT & VU B1ab(i,ii,iii)+2ab(i,ii,iii) & NT B1ab(iii)+2ab(iii) \\
\hline & Synallaxis cherriei & NT & NT A2c $+3 c$ & DD \\
\hline \multirow[t]{8}{*}{ Tyrannidae } & Polystictus pectoralis & NT & NT A2c+3c & VU A $2 c+3 c+4 c$ \\
\hline & $\begin{array}{l}\text { Pseudocolopteryx } \\
\text { acutipennis }\end{array}$ & $\mathrm{LC}$ & VU C1 & CR C2a(i) \\
\hline & Phylloscartes lanyoni* & $\mathrm{EN} \mathrm{A} 1 \mathrm{c}+2 \mathrm{c}$ & $\mathrm{EN} \mathrm{A} 3 \mathrm{c}+4 \mathrm{c}$ & EN C2a(i) \\
\hline & Aphanotriccus audax & NT & NT B1+2abce & NT B2ab(ii,iii,v) \\
\hline & Contopus cooperi & $\mathrm{LC}$ & LC & NT A2b+3b+4b \\
\hline & Muscisaxicola maculirostris & DD & EN B2ab(ii,iii) & EN B2ab(ii,iii,v) \\
\hline & Myiotheretes pernix* & VU C2b & EN B2ab(i,ii,iii,iv); C2a(ii) & EN B1ab(iii,v)+2ab(iii,v); C2a(ii) \\
\hline & Attila torridus & $\begin{array}{l}\text { VU A1c+2c; B1+2c; } \\
\mathrm{C} 1+2 \mathrm{a}\end{array}$ & $\begin{array}{l}\text { VU A4ce; B1ab(i,ii,iii,v)+2ab(i,ii,i } \\
\text { ii,v) }\end{array}$ & $\begin{array}{l}\text { VU A2c+4c; B1ab(ii, } \\
\text { iii,v)+2ab(ii,iii,v); C2a(i) }\end{array}$ \\
\hline \multirow[t]{10}{*}{ Cotingidae } & Pipreola chlorolepidota & NT & VU A4c & NT Blab(ii,iii,v) \\
\hline & Pipreola lubomirskii & NT & LC & LC \\
\hline & Ampelioides tschudii & NT & LC & LC \\
\hline & Doliornis remseni & $\mathrm{VU} A 1 \mathrm{c}+2 \mathrm{c} ; \mathrm{B} 1+2 \mathrm{c}$ & EN A4c; B2ab(ii,iii); C2a(i) & EN C2a(i) \\
\hline & Ampelion rufaxilla & $\mathrm{LC}$ & NT A3c & VU C2a(i) \\
\hline & Pyroderus scutatus & $\mathrm{LC}$ & LC & VU C2a(i) \\
\hline & Cephalopterus penduliger & VU C2a & VU A4c; C1+2(i) & EN C2a(i) \\
\hline & Lipaugus weberi*17 & N/A & CR C1 & CR C2a(i) \\
\hline & $\begin{array}{l}\text { Porphyrolaema } \\
\text { porphyrolaema }\end{array}$ & NT & LC & LC \\
\hline & Carpodectes hopkei & NT & LC & LC \\
\hline Pipridae & Chloropipo flavicapilla & NT & NT A3c & VU C2a(i) \\
\hline Tityridae & Laniisoma elegans & $\mathrm{VU} A 1 \mathrm{~b}+2 \mathrm{~b} ; \mathrm{C} 1+2 \mathrm{a}$ & LC & LC \\
\hline \multirow[t]{3}{*}{ Vireonidae } & Vireo masteri & VU D2 & VU B2ab(ii,iii) & VU B2ab(iii) \\
\hline & Vireo caribaeus* & $\mathrm{CR} B 1+2 \mathrm{bc}$ & CR B1ab(i,ii,iii)+2ab(i,ii,iii) & VU D2 \\
\hline & Vireo crassirostris* & $\mathrm{NE}$ & $\mathrm{NE}$ & VU D1+2 \\
\hline Corvidae & Cyanolyca pulchra & NT & NT A3c & VU C2a(i) \\
\hline Alaudidae & Eremophila alpestris & DD & EN B2ab(ii,iii,iv,v); C2a(i) & $\begin{array}{l}\text { EN A2ce+3ce+4ce; B2ab(i,ii,iii,iv,v); } \\
\text { C2a(i) }\end{array}$ \\
\hline \multirow[t]{3}{*}{ Troglodytidae } & Troglodytes monticola* 18 & N/A & N/A & EN B1ab(i,ii,iii,v); D \\
\hline & Cistothorus apolinari* & $\mathrm{EN} \mathrm{C} 1+2 \mathrm{a}$ & EN B2ab(ii,iii,iv,v); C2a(i) & CR B2ab(ii,iii,iv,v) \\
\hline & Thryophilus sernai* 19 & N/A & N/A & EN A3c+4c; B2ab(ii,iii,v); C2a(i) \\
\hline
\end{tabular}


Rev. Acad. Colomb. Cienc. Ex. Fis. Nat. 41(161):490-510, octubre-diciembre de 2017 doi: http://dx.doi.org/10.18257/raccefyn.461

\begin{tabular}{|c|c|c|c|c|}
\hline & Thryophilus nicefori* & $\mathrm{CR} \mathrm{B} 1+2 \mathrm{c}$ & CR B1ab(iii,v); C2(i,ii) & CR C2a(i) \\
\hline & Henicorhina negreti* 20 & N/A & N/A & VU B2ab(iii); C2a(i) \\
\hline \multirow[t]{2}{*}{ Turdidae } & Myadestes coloratus & $\mathrm{LC}$ & LC & VU B1ab(iii,v)+2ab(iii,v); C2a(ii) \\
\hline & Cichlopsis leucogenys & NT & LC & LC \\
\hline \multirow[t]{20}{*}{ Thraupidae } & Creurgops verticalis & LC & LC & VU C2a(i) \\
\hline & Thlypopsis ornata & LC & LC & NT B2ab(ii,iii,v); C2a(ii) \\
\hline & Bangsia melanochlamys* & $\mathrm{EN} \mathrm{B} 1 ; \mathrm{C} 1+2 \mathrm{a}$ & VU B2ab(iii) & VU B1ab(ii,iii,v) \\
\hline & Bangsia edwardsi & LC & LC & NT B1ab(ii,iii,v)+2ab(ii,iii,v) \\
\hline & Bangsia aureocincta* & $\mathrm{EN} \mathrm{B} 1+2 \mathrm{c} ; \mathrm{C} 1$ & EN B2ab(iii) & VU B1ab(ii,iii,v)+2ab(ii,iii,v) \\
\hline & Buthraupis wetmorei & $\begin{array}{l}\mathrm{VU} A 1 \mathrm{c}+2 \mathrm{c} ; \mathrm{B} 1+2 \mathrm{c} \\
\mathrm{C} 1+2 \mathrm{a}\end{array}$ & VU B1ab(ii,iii,v)+2ab(ii,iii,v) & VU B2ab(ii,iii,v) \\
\hline & $\begin{array}{l}\text { Iridosornis } \\
\text { porphyrocephalus }\end{array}$ & NT & NT A2c+3c & LC \\
\hline & Chlorochrysa nitidissima* & $\mathrm{VU} C 1+2 \mathrm{a}$ & VU A2b+3c; B2ab(ii,iii) & VU B2ab(ii,iii,v) \\
\hline & Tangara fucosa & NT & EN B1ab(ii,iii,v)+2ab(ii,iii,v); C2a(ii) & VU B1ab(iii,v)+2ab(iii,v); C2a(ii) \\
\hline & Tangara johannae & NT & $\mathrm{NT} \mathrm{A} 2 \mathrm{c}+3 \mathrm{c}$ & LC \\
\hline & Dacnis hartlaubi* & $\mathrm{VU} A 1 \mathrm{c} ; \mathrm{B} 1+2 \mathrm{c} ; \mathrm{C} 1+2 \mathrm{a}$ & VU B2ab(ii,iv); C2a(i) & VU B2ab(ii,iii,v); C2a(i) \\
\hline & Dacnis viguieri & NT & NT B1ab(iii) & NT B1ab(iii,v) \\
\hline & Dacnis berlepschi & VU C1 & VU A4c; B1ab(i,ii,iii)+2ab(i,ii,iii) & EN B1ab(ii,iii,v)+2ab(ii,iii,v); C2a(i) \\
\hline & Dacnis albiventris & NT & $\mathrm{LC}$ & LC \\
\hline & Oreomanes fraseri ${ }^{21}$ & NT & $\mathrm{DD}$ & $\mathrm{NE}$ \\
\hline & Diglossa gloriosissima* & NT & EN B2ab(ii,iii,iv) & VU B2ab(iii) \\
\hline & Saltator cinctus & VU C2a & VU A4c; B2ab(ii,iii) & VU B1ab(iii,v)+2ab(iii,v); C2a(i) \\
\hline & Sporophila insulata ${ }^{22}$ & CR D1 & CR B2ab(ii,iii,iv,v); C2a(i) & N/A \\
\hline & Sporophila crassirostris & NT & LC & LC \\
\hline & $\begin{array}{l}\text { Catamenia homochroa } \\
\text { oreophila }\end{array}$ & NT & $\mathrm{LC}$ & LC \\
\hline \multirow[t]{11}{*}{ Emberizidae } & Oreothraupis arremonops & VU C2a & VU B2ab(ii,iii,v) & LC \\
\hline & Chlorospingus tacarcunae & LC & LC & NT B1ab(iii,v)+2ab(iii,v);C2a(ii) \\
\hline & Chlorospingus flavovirens & VU C2a; D2 & VU B1ab(ii,iii,v); C1 & VU B1ab(iii)+2ab(iii); C2a(i) \\
\hline & Chlorospingus inornatus & LC & LC & VU B1ab(iii,v)+2ab (iii,v); C2a(ii) \\
\hline & Ammodramus savannarum & $\mathrm{EN} \mathrm{B} 1+2 \mathrm{c}$ & CR B2ab(iii); C1 & EN B2ab(i,ii,iii,iv,v); C2a(i) \\
\hline & Arremonops tocuyensis & $\mathrm{LC}$ & LC & NT A2c+4c; B1ab(ii,iii,v)+2ab(ii,iii,v) \\
\hline & Arremon schlegeli & LC & $\mathrm{LC}$ & VU B2ab(ii,iii,v); C2a(i) \\
\hline & Atlapetes flaviceps* & $\mathrm{EN} \mathrm{B} 1+2 \mathrm{ac} ; \mathrm{C} 2 \mathrm{a}$ & EN B2ab(ii,iii,v) & VU B2ab(ii,iii,v); C1 \\
\hline & Atlapetes fuscoolivaceus* & NT & VU B1ab(i,ii,iii,v)+2ab(i,ii,iii,v) & VU B1ab(ii,iii,v)+2ab(ii,iii,v) \\
\hline & Atlapetes leucopis & NT & LC & LC \\
\hline & Atlapetes blancae* 23 & N/A & N/A & CR-PE C2a(i); D \\
\hline \multirow[t]{2}{*}{ Cardinalidae } & Habia gutturalis & NT & NT B1+2abcde & LC \\
\hline & Cardinalis phoeniceus & $\mathrm{LC}$ & $\mathrm{LC}$ & VU A $2 c d+3 c d+4 c d$ \\
\hline \multirow[t]{5}{*}{ Parulidae } & Setophaga cerulea & LC & LC & $\mathrm{VU} A 2 b+3 b+4 b$ \\
\hline & Myiothlypis basilica* & NT & VU B1ab(i,ii,iii)+2ab(i,ii,iii) & EN B1ab(iii,v)+2ab(iii,v); C2a(i) \\
\hline & Myiothlypis conspicillata* & NT & EN B2ab(ii,iii) & VU B1ab(iii,v) \\
\hline & Myiothlypis cinereicollis & NT & NT A4c & NT B2ab(ii,iii,v) \\
\hline & Basileuterus ignotus & VU D2 & VU D1+2 & EN B1ab(iii,v)+2ab(iii,v); C2a(ii) \\
\hline \multirow[t]{2}{*}{ Icteridae } & Psarocolius cassini* & EN A2c & EN B2ab(ii,iii,v) & EN B2ab(ii,iii,v); C2a(i) \\
\hline & Cacicus uropygialis & VU C2a & NT A4c & DD \\
\hline
\end{tabular}




\begin{tabular}{|c|c|c|c|c|}
\hline & Icterus icterus & LC & LC & VU A2cd $+3 c d+4 c d ; C 1$ \\
\hline & Icterus leucopteryx & NE & $\mathrm{NE}$ & CR B1ab(iii,v) \\
\hline & Macroagelaius subalaris* & NT & CR B1ab(iii,v) & EN B2ab(i,ii,iii,iv,v); C2a(i) \\
\hline & $\begin{array}{l}\text { Hypopyrrhus } \\
\text { pyrohypogaster* }\end{array}$ & $\mathrm{EN} \mathrm{C} 1+2 \mathrm{a}$ & EN A4c & VU B2ab(i,ii,iii,iv,v); C2a(i) \\
\hline & Molothrus armenti* & DD & VU B1ab(i,ii,iii)+2ab(ii,iii) & VU B1ab(ii,iii,v)+2ab(ii,iii,v) \\
\hline Fringillidae & Spinus cucullatus & EN C2a & EN B1ab(iii,v)+2ab(iii,v); C2a(i) & $\begin{array}{l}\text { EN B1ab(ii,iii,v)+2ab(ii,iii,v); C2a(ii); } \\
\text { D }\end{array}$ \\
\hline
\end{tabular}

${ }^{1}$ Taxón endémico de Colombia. De la forma castaneus no se tenía certeza de su presencia en Colombia antes de 2006 (Laverde, 2014). Un estudio reciente de las vocalizaciones sugiere que la forma castaneus de la falla del Tequendama (cerca de Bogotá) podría ser considerada una especie distinta (Laverde, 2014). ${ }^{2}$ Crypturellus columbianus y C. saltuarius, antes consideradas especies separadas, no se evaluaron en 2016 porque ahora son consideradas subespecies de C. erythropus (Remsen, et al. 2016); ${ }^{3}$ Especie transeúnte (Remsen, et al. 2016); ${ }^{4}$ Era considerada una subespecie de Sula dactylatra lo cual le daba una distribución geográfica muy grande. La separación taxonómica hace que S. granti tenga una distribución muy restringida en el país (Pitman \& Jehl, 1998); ${ }^{5}$ Reconocida actualmente como una especie separada de A. floriceps (Lozano-Jaramillo, et al. 2014, Remsen, et al. 2016); ${ }^{6}$ Reconocidas actualmente como especies separadas de O. guerinii (Remsen, et al. 2016); ${ }^{7}$ Especie descrita después del año 2002 (Cortés-Diago, et al. 2007); ${ }^{8}$ Su estatus taxonómico era incierto, posteriormente se confirmó que es una especie válida (Krabbe, et al. 2005b, Remsen, et al. 2016); ${ }^{9}$ Antes Chaetocercus bombus. No se evaluó en 2016 porque no hay certeza de que existan poblaciones residentes en el país; ${ }^{10}$ Especie descrita después del año 2002 (Carantón \& Certuche, 2010 ); ${ }^{11}$ Reconocida como especie separada de S. femoralis (Remsen, et al. 2016); ${ }^{12}$ Especie descrita después del año 2002 (Krabbe, et al. 2005a); ${ }^{13}$ Especie descrita después del año 2002 (Cuervo, et al. 2005); ${ }^{14}$ Especie descrita después del año 2002 (Avendaño, et al. 2015); ${ }^{15}$ Reconocida actualmente como especie separa de S. opacus (Remsen, et al. 2016); ${ }^{16}$ Reconocida actualmente como especie separa de C. rubiginosus (Remsen, et al. 2016); ${ }^{17}$ Especie descrita después del año 1998 (Cuervo, et al. 2001); ${ }^{18}$ Reconocida como especie separada de T. solstitialis (Remsen, et al. 2016); ${ }^{19}$ Especie descrita después del año 2002 (Lara, et al. 2012); ${ }^{20}$ Especie descrita después del año 2002 (Salaman, et al. 2003); ${ }^{21}$ Especie con menos del 2\% de su población en el país. No evaluada en 2016 por criterio de filtrado; ${ }^{22}$ Antes se consideraba una especie válida, hoy se sabe que es un híbrido entre S. telasco y S. minuta (De las Casas, et al. 2004); ${ }^{23}$ Especie descrita como nueva para la ciencia después del año 2002 (Donegan, 2009).

emprendimientos turísticos y las oportunidades de empleo que estos últimos generan (Isaac, et al., 2004, Garnett \& Christidis, 2017). Estas dificultades han llevado a algunos autores a proponer una mejor gobernanza de la taxonomía de organismos complejos mediante un sistema de estándares para definir los límites de especies y otras unidades taxonómicas que pueda aplicarse confiablemente a diferentes formas de vida (Garnett \& Christidis, 2017). Otros autores han hecho la recomendación de usar una lista estandarizada (por ejemplo, una lista de chequeo de sinonimias) como un punto de referencia que, además, facilitaría el manejo de la biodiversidad, pues las prioridades de conservación suelen basarse en listas de especies y es importante que estas sean relativamente estables (Isaac, et al., 2004). Esta discusión se encuentra lejos de haberse dirimido y amerita ser abordada por los científicos dedicados a la sistemática y la taxonomía dadas las implicaciones que tiene más allá de esta área específica de investigación.

Otros cambios. Algunas especies ya analizadas en el pasado no han sido evaluadas recientemente a escala nacional. Pterodroma phaeopygia (CR en 2002) y Chaetocercus bombus (VU en 2002) no se han evaluado recientemente porque no hay evidencia de que haya poblaciones residentes en Colombia y los registros notificados podrían tratarse de individuos errantes. Oreomanes fraseri (DD en 2002) no se ha evaluado tampoco porque se estima que su población en el país es de menos del $2 \%$ de la población global.

Algunos cambios de categoría no pueden clasificarse como mejoría (downlist), empeoramiento (uplist), o como sin cambios, y ameritan explicación. Dos especies de colibríes pasaron de DD a CR-PE: la primera, Heliangelus zusii, se categorizó como DD en 2002 porque se conocía exclusivamente de un espécimen recolectado en 1909 sin localidad exacta y no había certeza de que fuera una especie válida (Renjifo, et al., 2002). En estudios más recientes se sugiere que esta especie es válida y que pertenece a un grupo de colibríes que está asociado a enclaves secos en la cordillera de los Andes (Kirchman, et al., 2009, Parra, 2016), y aunque es probable que esté extinta, no hay certeza de que así sea (Renjifo, et al., 2016). No obstante, la evidencia ya recolectada pero sin publicar sugiere que el único espécimen conocido podría tratarse de un híbrido (Pérez-Emán, et al., en preparación), lo cual enfatiza las dificultades que genera la inestabilidad taxonómica para la conservación (Isaac, et al., 2004). La segunda, Eriocnemis godini, se consideró DD en el año 2002 porque solo se conocían dos pieles comerciales del siglo XIX y no existía evidencia inequívoca de que hubiese habitado en Colombia (Renjifo, et al., 2002). Actualmente se considera CR-PE porque, aunque es probable que esté extinta, no hay certeza de ello. Considerando la similitud ecológica y la cercanía de los enclaves secos montanos donde se encontraba esta especie en Ecuador y los enclaves secos montanos de Nariño y Cauca, cabe la posibilidad de que exista en ellos una población remanente (Renjifo, et al., 2016).

Por otro lado, Cypseloides lemosi se consideró CR en 2002 por la ausencia de registros durante casi diez años en los valles altos del Cauca y el Patía en los departamentos de Valle del Cauca y Cauca, una región frecuentada por ornitólogos y observadores de aves (Renjifo, et al., 2002). Más aún, la especie se consideraba endémica de Colombia en los valles del alto Cauca y el alto Patía (Hilty \& Brown, 1986), posteriormente fue encontrada en el piedemonte amazónico de Perú y Ecuador y después del 2002 en el sur del 
piedemonte amazónico en Colombia (Ayerbe-Quiñones, 2016). Aunque el aumento de información significa una ampliación de la distribución de la especie, el escaso conocimiento de sus requerimientos ecológicos impide concluir con certeza si se encuentra amenazada (Renjifo, et al., 2016).

Por último, dos especies consideradas NT en 2002 hoy se consideran DD. La primera de ellas, Synallaxis cherriei, se consideró como tal en 2002 según el criterio A y con base en la estimación de BirdLife International (2000) (Renjifo, et al. 2002), pero actualmente no se acerca a los umbrales de amenaza bajo este criterio y no es evaluable aplicando los criterios B2, C y D porque no hay suficiente información para estimar el área de ocupación ni la población de la especie aplicable a Colombia (Renjifo, et al., 2016). La segunda especie que cambió de NT a DD es Cacicus uropygialis. En el 2002 se la consideró NT según el criterio A (Renjifo, et al., 2002), pero actualmente, la falta de claridad en torno a los registros visuales dada su similitud, impide que puedan utilizarse en el modelamiento de la distribución de la especie, pues los datos que se obtendrían no serían suficientemente confiables (Renjifo, et al., 2014).

Evolución en los criterios de amenaza. El criterio más prevalente bajo el cual las especies colombianas califican como amenazadas es el criterio B (70\% de las especies en 2002 y $72 \%$ en 2016), es decir especies con distribuciones pequeñas, seriamente fragmentadas y en disminución. El segundo criterio es el C ( $44 \%$ de las especies en 2002 y 66 $\%$ en 2016), es decir especies con poblaciones pequeñas y en disminución. En tercer lugar se encuentra el criterio A (38 \% en 2002 y $24 \%$ en 2016), o sea, especies que experimentan una rápida reducción de su tamaño poblacional. Por último está el criterio D ( $13 \%$ en 2002 y $25 \%$ en 2016), es decir, especies con población o una distribución muy pequeña, y bajo amenaza natural o antrópica plausible (Tabla 2). Mientras que el criterio B se ha mantenido estable (ha aumentado en $1,4 \%$ ), la proporción de especies amenazadas bajo los otros criterios ha cambiado dramáticamente. El criterio A disminuyó en $36,8 \%$, los criterios $\mathrm{C}$ y $\mathrm{D}$ aumentaron en $43,5 \%$ y $92,3 \%$, respectivamente. Estos cambios se deben tanto a los que se han producido en los procesos que afectan las especies como a mejoras importantes en la disponibilidad de información y en los métodos analíticos.

No se obtuvieron estimaciones del tamaño poblacional de 36 de las especies que estaban en alguna categoría de amenaza en el 2002 (32\% del total). De estas 36 especies, 28 seguían estando amenazadas en el 2016, y para cinco de ellas ya se cuenta con estimaciones del tamaño poblacional; las 23 restantes persisten como especies cuyo tamaño poblacional no es posible estimar. Asimismo, para 86 de las 140 especies actualmente amenazadas (61\%) no existe información que permita la estimación de su tamaño poblacional a partir de la densidad poblacional de la especie (Tabla 3S, https://www.raccefyn.co/index.php/raccefyn/article/ downloadSuppFile/461/2390), lo cual debe incentivar a los ornitólogos de campo a investigar la densidad poblacional de especies amenazadas y casi amenazadas en diferentes tipos de ecosistemas. Sin esa información no se puede saber si se encuentran amenazadas según los criterios C y D.

\section{Una mirada global del estado de conservación actual de la avifauna colombiana}

De las 1.786 especies residentes en Colombia permanentemente o durante una época del año, una está extinta, $140(7,9 \%)$ están amenazadas (CR: 17; EN: 56, y VU: $67)$ y 28 especies $(1,6 \%)$ están casi amenazadas (Tabla 4S, https://www.raccefyn.co/index.php/raccefyn/article/ downloadSuppFile/461/2391). En Colombia hay 84 familias de aves residentes, de las cuales 50 tienen cinco o más especies. Entre estas últimas, las familias Psittacidae y Grallariidae se encuentran más amenazadas de lo que podría esperarse, y Cracidae muestra una clara tendencia en ese sentido (prueba exacta de Fisher: $p<0,05$ ) (Tabla 3). De las 11 especies de psitácidos que están amenazadas en Colombia, seis lo están por tenencia como mascotas, cinco por cacería, dos por tráfico ilegal y cinco por expansión de la frontera agropecuaria. Las siete especies de la familia Grallariidae que están amenazadas, lo están por pérdida de hábitat, especialmente por la expansión de la frontera agropecuaria. Las especies de la familia Cracidae que se encuentran amenazadas lo están tanto por la pérdida de hábitat como por la cacería.

De las 140 especies amenazadas en Colombia, 61 se encuentran principalmente en los bosques húmedos de los Andes, 20 en los ecosistemas abiertos o secos (sabanas, páramos, bosques secos, matorral espinoso y desierto) y 16 en los bosques húmedos de las tierras bajas en el Pacífico, en el centro y norte del país, y en el piedemonte al oriente de los Andes. Además, 15 especies amenazadas son acuáticas y se encuentran en diversas partes del país, y tres son marinas, dos de las cuales se reproducen solo en la isla de Malpelo. Cinco especies son insulares, cuatro de ellas se encuentran únicamente en San Andrés y Providencia (o solo en una de estas dos islas), y una especie es de las islas de los grandes ríos amazónicos (Caquetá y Amazonas y, probablemente, Putumayo).

Las regiones más críticas por el número de especies amenazadas y con distribución restringida, son la Sierra Nevada de Santa Marta, con 11 especies endémicas amenazadas, y las montañas del Darién, con nueve especies. Si bien todas las especies de la Sierra Nevada se encuentran en áreas pertenecientes al sistema de parques nacionales naturales, ninguna de las del Darién lo está. La gravedad de la situación de las especies de las montañas del Darién se ha agudizado por la reciente expansión de los cultivos ilícitos de coca en el norte del Chocó y el hecho de que estos sistemas montañosos son tan pequeños que ni siquiera son visibles en la escala en que aparecen las otras áreas aquí mencionadas (Figura 1). Tanto las montañas del Darién como la Sierra Nevada albergan, además de sus especies endémicas amenazadas, otras cuya distribución se encuentra 
Tabla 3. Distribución de especies amenazadas entre las familias (prueba exacta de Fisher), tras excluir las especies DD. Los asteriscos representan las especies para las cuales la proporción de amenaza observada está por encima de la proporción de amenaza esperada (valor $\mathrm{p}<0,05$ ).

\begin{tabular}{lcccc|}
\hline Familia & $\begin{array}{c}\text { Especies } \\
\text { amenazadas }\end{array}$ & $\begin{array}{c}\text { Especies no } \\
\text { amenazadas }\end{array}$ & Total & (valor $\boldsymbol{p})$ \\
\hline Tinamidae & 3 & 14 & 17 & 0,300 \\
\hline Anatidae & 6 & 13 & 19 & 0,116 \\
\hline Cracidae & 7 & 18 & 25 & 0,069 \\
\hline Odontophoridae & 3 & 7 & 10 & 0,291 \\
\hline Sulidae & 2 & 3 & 5 & 0,222 \\
\hline Ardeidae & 1 & 22 & 23 & 0,883 \\
\hline Threskiornithidae & 0 & 7 & 7 & 1,000 \\
\hline Cathartidae & 1 & 5 & 6 & 0,500 \\
\hline Accipitridae & 3 & 46 & 49 & 0,782 \\
\hline Rallidae & 2 & 25 & 27 & 0.694 \\
\hline Charadriidae & 0 & 9 & 9 & 1,000 \\
\hline Scolopacidae & 0 & 29 & 29 & 1,000 \\
\hline Laridae & 1 & 17 & 18 & 0.757 \\
\hline Columbidae & 3 & 32 & 35 & 0.663 \\
\hline Cuculidae & 1 & 17 & 18 & 0.757 \\
\hline Strigidae & 1 & 23 & 24 & 0.883 \\
\hline Nyctibiidae & 0 & 5 & 5 & 1,000 \\
\hline Caprimulgidae & 0 & 21 & 21 & 1,000 \\
\hline Apodidae & 0 & 14 & 14 & 1,000 \\
\hline Trochilidae & 13 & 149 & 162 & 0.580 \\
\hline Trogonidae & 0 & 15 & 15 & 1,000 \\
\hline Alcedinidae & 0 & 6 & 6 & 1,000 \\
\hline Momotidae & 0 & 6 & 6 & 1,000 \\
\hline
\end{tabular}

mayoritariamente en los Andes o en los bosques húmedos de tierras bajas. Por último, los bosques de la gran llanura amazónica no tienen especies amenazadas, salvo la especie de las islas ya mencionada.

Las causas de amenaza que enfrentan las especies son diversas. El $55 \%$ está afectado por la agricultura y el 44,3\%, por la ganadería, $32,1 \%$ de ellas por la cacería y el tráfico ilegal, $31,4 \%$ por los cultivos ilícitos, $27,9 \%$ por cambios en los sistemas naturales, $22,9 \%$ por la explotación forestal, $19,3 \%$ por la producción de energía y minería, $16,4 \%$ por especies invasoras, $15,7 \%$ por el transporte y los corredores de servicio, $12,1 \%$ por el desarrollo residencial y comercial, $10 \%$ por la contaminación, $5 \%$ por los fenómenos climáticos y 3,6 \% por la intrusión de humanos (Figura 2; Tabla 4). El $80 \%$ de las especies amenazadas lo está por más de una de estas causas, y $98 \%$ de ellas han perdido su hábitat.

\section{Discusión}

Nuestros resultados indican que en las evaluaciones de riesgo de extinción se utilizó de manera implícita o explícita el sistema de la UICN vigente en su momento. Las dos

\begin{tabular}{|c|c|c|c|c|}
\hline Galbulidae & 1 & 13 & 14 & 0.759 \\
\hline Bucconidae & 0 & 24 & 24 & 1,000 \\
\hline Capitonidae & 2 & 6 & 8 & 0,500 \\
\hline Ramphastidae & 2 & 19 & 21 & 0,697 \\
\hline Picidae & 2 & 39 & 41 & 0,820 \\
\hline Falconidae & 1 & 16 & 17 & 0,758 \\
\hline Psittacidae ${ }^{* * *}$ & 11 & 43 & 54 & 0,046 \\
\hline Thamnophilidae & 3 & 110 & 113 & 0,983 \\
\hline Grallariidae*** & 13 & 32 & 45 & 0,015 \\
\hline Formicariidae & 0 & 8 & 8 & 1,000 \\
\hline Furnariidae & 3 & 107 & 110 & 0,983 \\
\hline Tyrannidae & 6 & 195 & 201 & 0,993 \\
\hline Cotingidae & 5 & 28 & 33 & 0,354 \\
\hline Pipridae & 1 & 19 & 20 & 0,885 \\
\hline Tityridae & 0 & 20 & 20 & 1,000 \\
\hline Vireonidae & 3 & 18 & 21 & 0,500 \\
\hline Corvidae & 1 & 6 & 7 & 0,769 \\
\hline Hirundinidae & 0 & 17 & 17 & 1,000 \\
\hline Troglodytidae & 5 & 28 & 33 & 0,354 \\
\hline Polioptilidae & 0 & 6 & 6 & 1,000 \\
\hline Turdidae & 1 & 27 & 28 & 0,882 \\
\hline Thraupidae & 10 & 162 & 172 & 0,855 \\
\hline Emberizidae & 7 & 29 & 36 & 0,154 \\
\hline Cardinalidae & 1 & 21 & 22 & 0,884 \\
\hline Parulidae & 4 & 38 & 42 & 0,500 \\
\hline Icteridae & 6 & 33 & 39 & 0,240 \\
\hline Fringillidae & 1 & 21 & 22 & 0,884 \\
\hline Total & 136 & 1588 & 1724 & \\
\hline
\end{tabular}

primeras (Rodríguez-Mahecha, et al., 1986, Negret, 2001) utilizaron un sistema de categorías, mientras que las últimas tres han utilizado un sistema de categorías y criterios (Renjifo, 1998, Renjifo, et al., 2002, Renjifo, et al., 2016). Es preciso señalar que si bien el estudio de Negret (2001) fue publicado después del de Renjifo (1998), la evaluación se hizo previamente, cuando aún no se había adoptado el sistema de criterios de la UICN. Las tres primeras evaluaciones se basaron en el criterio experto (RodríguezMahecha, et al., 1986, Negret, 2001, Renjifo, 1998), en tanto que las dos últimas utilizaron métodos innovadores para la estimación de los parámetros que sirvieron para las estimaciones cuantitativas sobre cada especie, aunque en las dos se recurrió también al criterio experto (Renjifo, et al., 2002, Renjifo, et al., 2016). Estos nuevos métodos incluyen el uso de sistemas de información geográfica y la estimación del tamaño poblacional con base en el conocimiento de las especies o de especies estrechamente emparentadas o ecológicamente similares. En la última evaluación se utilizó la modelación de nicho ecológico de las especies para 


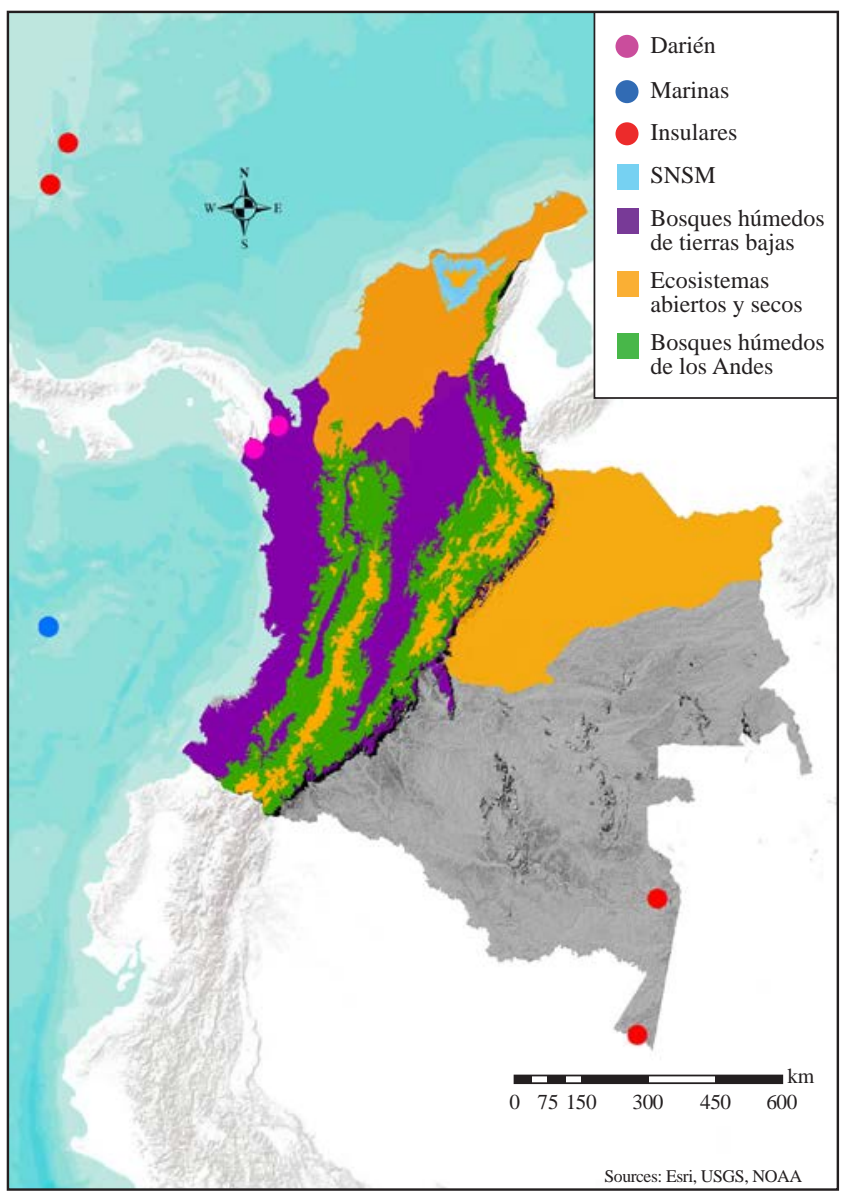

Figura 1. Ubicación de las áreas de distribución de las especies de aves amenazadas en Colombia enumeradas en el cuerpo del artículo. Los ecosistemas abiertos o secos agrupan las sabanas, los páramos, los bosques secos, las áreas de matorral espinoso y el desierto. La geografía de las especies acuáticas no está representada, pues está dispersa en diferentes regiones del país y su distribución se traslapa con las regiones representadas en el mapa. estimar el tamaño de la distribución geográfica y el área de ocupación. Asimismo, se utilizó información multitemporal del área de los ecosistemas para medir el ritmo de cambio en la extensión de los hábitats de las especies e inferir la tendencia y la magnitud del cambio en el tamaño de las poblaciones.

En Colombia el número de especies amenazadas de aves se ha incrementado progresivamente, lo cual sugiere un acentuamiento de la magnitud y la intensidad de las amenazas y un mayor conocimiento de las especies. A partir del 2002 el conocimiento sobre la distribución, la ecología y la sistemática de aves colombianas ha venido aumentando, como lo destacan varias publicaciones (Bravo \& Naranjo, 2006, Estela, et al., 2010, Naranjo \& Bravo, 2006, Peñuela-Ocampo, 2010). En la medida en que se incrementa el número de especies conocidas en el país también crece el de las especies potencialmente amenazadas, las cuales se suman a las que han tenido un deterioro genuino de su estado poblacional.

Nuestros resultados evidencian que actualmente las principales amenazas para las aves son la expansión de la frontera agropecuaria, los cultivos ilícitos y la minería. La situación ambiental del país ha cambiado profundamente en las últimas tres décadas. Los conflictos ambientales han aumentado considerablemente desde el 2002 y hay evidencia de que este aumento está relacionado con los cultivos ilícitos y las políticas que promueven la inversión extranjera en el sector extractivo del país. Entre 1990 y 2001 se registraron diez conflictos ambientales en Colombia, en tanto que entre el 2002 y el 2010 se reportaron 47 (Pérez-Rincón, 2014). Actualmente, el proyecto Environmental Justice Organizations, Liabilities and Trade (EJOLT), conocido en español como Atlas Global de Justicia Ambiental, ha recabado datos que evidencian cómo Colombia registró 116 casos de conflictos socio-ambientales en abril de 2016 (EJOLT

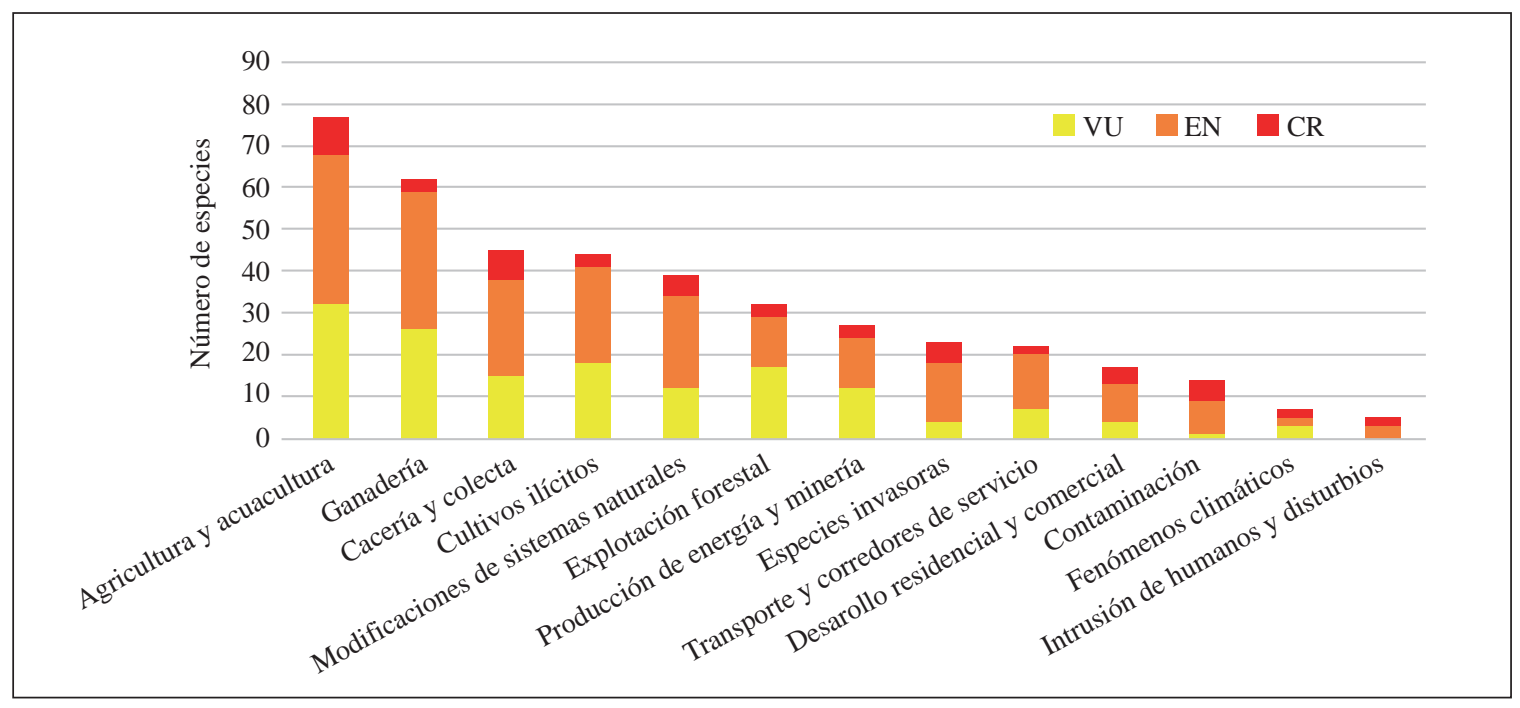

Figura 2. Amenazas directas para las aves clasificadas como amenazadas en Colombia 
Tabla 4. Amenazas directas sobre las especies de aves en Colombia basada en la clasificación de tipos de amenazas de la UICN (World Conservation Union-Conservation Measures Partnership (IUCN$C M P$ ) classification of direct threats to biodiversity, version 1.1) y modificada para incluir la categoría de cultivos ilícitos. Usualmente una especie es afectada por más de un factor de amenaza.

\begin{tabular}{|lc|}
\hline Factor de amenaza & $\begin{array}{c}\text { Número de especies } \\
\text { afectadas }\end{array}$ \\
\hline 1. Desarrollo residencial y comercial & 10 \\
\hline Expansión urbana & 2 \\
\hline Desarrollo comercial e industrial & 6 \\
\hline Turismo y áreas recreacionales & \\
\hline 2. Agricultura y acuacultura & 3 \\
\hline Pesquerías & 77 \\
\hline Cultivos y plantaciones & 62 \\
\hline Ganadería & 44 \\
\hline Cultivos ilícitos & \\
\hline
\end{tabular}

\section{Producción de energía y minería}

Actividades petroleras 4

Minería

24

\section{Transporte y corredores de servicio}

Carreteras y vías

19

Colisiones/electrocución con infraestructura

eléctrica

\begin{tabular}{|c|c|}
\hline 5. Uso de recursos biológicos & \\
\hline Cacería y colecta & \\
\hline Tráfico ilegal & 39 \\
\hline Cacería de subsistencia & 10 \\
\hline Tenencia como mascota & 13 \\
\hline Explotación forestal & 31 \\
\hline 6. Intrusión y disturbios por humanos & 5 \\
\hline 7. Modificaciones de sistemas naturales & \\
\hline
\end{tabular}

Quemas inducidas o cambios en la frecuencia de fuegos

\begin{tabular}{lr} 
Construcción y operación de embalses & 5 \\
\hline Drenajes de humedales & 10 \\
\hline Pérdida de fuentes de agua & 1
\end{tabular}

\section{Especies invasoras y otras problemáticas}

\begin{tabular}{|lc|}
\hline $\begin{array}{l}\text { Animales invasores no nativos (perros, gatos, } \\
\text { ratas, cabras y truchas) }\end{array}$ & 19 \\
\hline Plantas invasoras & 6 \\
\hline $\begin{array}{l}\text { Parasitismo de cría por el Chamón Molothrus } \\
\text { bonariensis }\end{array}$ & 3 \\
\hline 9. Contaminación & 14 \\
\hline 10. Fenómenos climáticos & 7 \\
\hline
\end{tabular}

Project, 2016), es decir más del doble de los ocurridos seis años atrás. La extracción de minerales y la producción de petróleo se destacan entre los motivos de dichos conflictos ambientales. La actividad minera ha motivado el $42 \%$ de los conflictos, la energía fósil, el $19 \%$ y la biomasa, el $10 \%$ (especialmente los cultivos de palma y caña) (Pérez-Rincón, 2014, según datos de Univalle-EJOLT-Colombia). En los años noventa la exportación de petróleo y sus derivados representó el $30 \%$ del valor de las ventas en exportaciones, en el 2012 representaban más de $50 \%$ y en los últimos años, el sector minero-energético generó el $64 \%$ de los productos exportados por Colombia (Pérez-Rincón, 2014). Por otro lado, en algunas regiones han aparecido nuevas fuentes de impacto, como los cultivos de palma de aceite destinados a la elaboración de biocombustible (Arboleda, 2008).

La magnitud y la intensidad de las amenazas directas sobre la avifauna dependen también de actividades ilegales ampliamente extendidas en el país. Según la Contraloría General, la minería ilegal, por ejemplo, representaba en el 2012, el $80 \%$ de la actividad minera del país (Contraloría General de la República, 2012). Por otra parte, después de la ganadería, la segunda causa de deforestación en Colombia es la tala ilegal (García, 2014). El Banco Mundial estimó en el 2006 que la tala ilegal de madera correspondía a $42 \%$ del total de la producción de madera en Colombia (Banco Mundial, 2006). Por otro lado, aunque el comercio y la tenencia de especies amenazadas es una actividad tipificada como delito en la normatividad ambiental (Decreto 1608 de 1978) y en la penal (Ley 599 de 2000), estas actividades han alcanzado niveles muy significativos en Colombia. Según Convención sobre el Comercio Internacional de Especies Amenazadas de Fauna y Flora Silvestres (CITES, 2012), entre el 2000 y el 2011 se intentaron exportar de manera ilegal 3.744 especímenes vivos y 24.985 derivados de especies (Rojas-Briñez, et al., 2013). La persistencia de estas actividades ilegales que amenazan a las aves es consecuencia de la falta de control del Estado colombiano en los territorios, donde dichas actividades representan la principal amenaza para las aves del país. Estas amenazas tomadas en conjunto sugieren que la principal causa de amenaza para las aves es la falta de gobernabilidad del Estado sobre el territorio.

\section{Evolución de los criterios de amenaza}

La magnitud de la amenaza contra las especies colombianas con distribuciones pequeñas, seriamente fragmentadas y en disminución (criterio B), es la manifestación de diversos factores. En primer lugar, responde al gran número de especies con distribución restringida presentes en Colombia comparado con el de otras regiones del mundo (Sttatersfield, et al., 1998), y, en segundo lugar, indica el alcance de la destrucción de los hábitats en algunas regiones del país. Esta destrucción afecta especialmente a las especies con baja tolerancia a la transformación del paisaje. Las especies de distribución restringida son doblemente vulnerables, tanto por el tamaño de su distribución, como por ser más sensibles localmente a la transformación del paisaje, en comparación con las especies de amplia distribución (Renjifo, 1999).

Mientras que la proporción de especies amenazadas bajo el criterio $\mathrm{B}$ se ha mantenido relativamente estable (solo ha aumentado en 1,4 \% entre 2002 y 2016), la proporción 
de las amenazadas bajo los otros criterios ha cambiado dramáticamente. Si bien los métodos utilizados para evaluar el criterio B fueron diferentes en el 2002 y el 2016, los resultados concordaron en gran medida. En el 2002 las estimaciones del tamaño de la distribución de las especies se basaron en el criterio de expertos y en el 2014 y el 2016, en el modelamiento de nicho y en el criterio experto.

Debe destacarse que no todas las especies se evaluaron según todos los criterios. El nivel de conocimiento sobre la distribución de las especies es superior comparado con el conocimiento sobre el tamaño de las poblaciones y el cambio en la cobertura de los hábitats de las especies. Mientras el $100 \%$ de las especies amenazadas y casi amenazadas fueron evaluables según el criterio B y el 'subcriterio' D2 (ambos basados en tamaños de la distribución), el $12 \%$ de las especies amenazadas en el 2016 no pudo evaluarse según el criterio $\mathrm{C}$ por carecer del sustento suficiente para hacer inferencias sobre su tamaño poblacional, esto a pesar de que la disponibilidad de información sobre algunas especies mejoró comparada con la disponibles para la evaluación del 2002.

Los métodos para la estimación de los tamaños poblacionales en lo relacionado con la evaluación del criterio C fueron similares en el 2002 y el 2016. Por el contrario, los métodos para evaluar el criterio A fueron totalmente diferentes. El principal factor tomado en cuenta para estimar la reducción o el aumento del tamaño poblacional de las especies (criterio A) en Colombia ha sido el cambio en la extensión de los hábitats de cada especie. Estas estimaciones se basaron en el criterio experto en la evaluación del 2002 y en el uso de sistemas de información geográfica e información multitemporal de la cobertura de los ecosistemas del país en la evaluación del 2016.

La proporción de especies amenazadas según el criterio A disminuyó en 36,8 \% en el 2016 comparado con el 2002. Las evaluaciones para el criterio A difirieron en cuanto a los métodos entre el 2002 y el 2016. Los cambios en la categoría de las especies bajo este criterio son atribuibles a los avances metodológicos en la estimación de la tasa de pérdida de hábitat $\mathrm{y}$, especialmente, a los profundos cambios en las dinámicas de pérdida y regeneración de ecosistemas en diversas regiones del país. Durante décadas, en diversas fuentes se ha resaltado la rápida pérdida de bosques en diferentes escalas tanto espaciales como temporales (Etter, et al., 2006, Cabrera, et al., 2011, Dávalos, et al., 2011). No obstante Sánchez-Cuervo, et al. (2012) encontraron "tendencias sorprendentes de recuperación forestal entre el 2001 y el 2010”. Esta recuperación forestal es el resultado del abandono de tierras como consecuencia de varios factores que incluyen la falta de oportunidades económicas y el conflicto armado, así como los programas de erradicación de coca que llevaron a una drástica reducción de estos cultivos en la primera década de este siglo (Programa de Naciones Unidas para el Desarrollo, 2011, Sánchez-Cuervo, et al., 2012). Nuestros datos, obtenidos de manera independiente en el proceso de análisis, indican precisamente ese patrón al evaluar algunas de las especies. La recuperación de bosques en algunas regiones y la reducción en el ritmo de la deforestación en otras han permitido que algunas especies hayan pasado a categorías de menor riesgo y que, incluso, algunas hayan dejado de estar amenazadas (por ejemplo, Coeligena prunellei).

No obstante, estas tendencias distan de ser homogéneas a través del país. Los cultivos de coca han sido un factor importante en la deforestación del país (Dávalos, et al., 2009). Los centros de producción de coca han cambiado sustancialmente en lo que va corrido de este siglo. La intensificación de los programas de erradicación y el aumento de las amenazas sobre los productores de coca han promovido el desplazamiento de los cultivos ilícitos hacia áreas con baja presencia estatal (Dion \& Russler, 2008, Dávalos, et al., 2009). Los ecosistemas de la región pacífica se vieron mucho más afectados por los cultivos de coca entre el 2001 y el 2008 (Rincón-Ruiz, et al., 2013). Las campañas de erradicación en Putumayo han desplazado los cultivos a Nariño, departamento donde antes no se habían registrado cultivos de coca (Dávalos, et al., 2009). En consecuencia, especies previamente amenazadas por la rápida deforestación de los Andes en Putumayo y Caquetá a finales del siglo pasado, han visto reducida su categoría de amenaza cuando los cultivos se trasladaron a la región del Pacífico, en especial, a Nariño (por ejemplo, Pipreola chlorolepidota). El aumento de los cultivos ilícitos en la región pacífica, y en particular en Nariño, ha tenido un grave efecto sobre otro conjunto de especies con distribuciones muy pequeñas. Andigena laminirostris, Capito squamatus y Dacnis berlepschi pasaron a mayores niveles de amenaza. En el caso de Micrastur plumbeus y Campephilus gayaquilensis la situación empeoró de modo aún más dramático al pasar de casi amenazadas a estar en peligro. Los más recientes incrementos en el área cultivada de coca en el país nos obligan a ser pesimistas sobre el estado de un conjunto importante de especies.

El mayor grado de amenaza para las familias Psittacidae, Grallariidae y Cracidae, respecto a las demás familias, coincide con las tendencias regionales o globales. Los psitácidos son el grupo de aves con más especies amenazadas en el mundo, pues $27 \%$ de las 374 especies existentes lo están (Snyder, et al., 2000, Parr \& Juniper, 1998, IUCN, 2015). Las principales causas de amenaza son la pérdida de hábitat y su fragmentación, así como la explotación de sus poblaciones (Snyder, et al., 2000), tal como pudimos constatarlo en este estudio a nivel nacional. Por otra parte, los insectívoros terrestres, como las especies del género Grallaria, tienden a extinguirse en paisajes fragmentados (Renjifo, 1999), lo cual es congruente con el hecho de que, según nuestros datos, la principal causa de amenaza para esta familia ha sido la expansión de la frontera agropecuaria. Los crácidos, por su parte, se consideran la familia de aves más amenazada de las Américas tanto por la deforestación como por la cacería (Brooks, et al., 2006). 
Como se ha señalado en el desarrollo de este artículo, las listas y libros rojos proveen información orientada a evitar la extinción de especies. Sin embargo, es preciso señalar que tienen una importante limitación: la ausencia de evaluaciones de riesgo de subespecies o de poblaciones regionales. El desarrollo de este tipo de evaluaciones se enfrenta con limitaciones importantes en cuanto a conocimientos y prácticas, entre ellas, la escasa información sobre la validez de estos taxones, los límites de sus distribuciones y las diferencias en cuanto a requerimientos ecológicos, etc., por lo que, en caso de ser evaluadas, un número muy importante quedaría en la categoría de "datos insuficientes" (DD). No obstante, es evidente que algunas subespecies ya se han extinguido, como es el caso de Anas georgica niceforoi (Rodríguez-Mahecha, et al., 1986) y otras se encuentran al borde de la extinción, como la garcita Ixobrychus exilis bogotensis, endémica de los humedales del altiplano cundiboyacense (Renjifo, et al., 2016). Esta situación resalta la necesidad de desarrollar iniciativas de conservación de algunas subespecies a escala regional.

Una limitación importante del sistema de criterios de la UICN es que una mejoría de categoría según los criterios $\mathrm{B}, \mathrm{C}$ y D implica una mejoría en el estado de la especie en cuanto al tamaño de su distribución o de su población, pero esto no ocurre con un cambio de categoría basado en el criterio A. Este criterio evalúa la proporción de la reducción de la población de una especie, así que que cuando una especie pasa a una categoría de menor riesgo según este criterio, ello no implica una recuperación del tamaño poblacional sino una reducción en el ritmo de pérdida de población. En otras palabras, la especie no necesariamente ha mejorado, sino que está empeorando más lentamente.

Por último, para futuras evaluaciones quisiéramos enfatizar la importancia de la obtención de información valiosa en torno a la densidad de las poblaciones de las especies, preferiblemente en diferentes tipos de hábitats y en sus gradientes de idoneidad. También debe garantizarse que los registros y la precisión de la georreferenciación en el momento de la recolección de datos, así como la exploración de sitios sobre los cuales hay poca información ornitológica, sean óptimos.

\section{Agradecimientos}

Esta investigación se desarrolló gracias al apoyo económico y logístico de la Vicerrectoría de Investigación y la Facultad de Estudios Ambientales y Rurales de la Pontificia Universidad Javeriana. Estamos agradecidos con Nicolás Urbina por sus valiosas sugerencias, y con Nick Bayly y Felipe Estela, por compartir con nosotros su conocimiento sobre la condición migratoria o accidental de especies en San Andrés y Providencia, y de especies marinas. Nuestros agradecimientos también van para Alberto Ramírez y Néstor Peralta, por sus opiniones sobre las pruebas estadísticas y, de manera especial, a Juan Carlos Benavides, quien nos asesoró en el cálculo de la prueba exacta de Fisher. Asimismo, agradecemos a Jaime Burbano-Girón la elaboración del mapa de la Figura 2. Los comentarios de Carlos Daniel Cadena y de los dos revisores anónimos contribuyeron a ajustar el contenido del artículo.

\section{Información suplementaria}

Tabla 1S. Especies y subespecies de aves de Colombia amenazadas en 1986 según Rodríguez-Mahecha, et al. 1986. Los nombres de las especies se actualizaron de acuerdo con SACC (2016), pero se conserva la clasificación original de las familias de ese listado. Vea la tabla $1 \mathrm{~S}$ en: https://www.raccefyn.co/index.php/raccefyn/article/ downloadSuppFile/461/2388

Tabla 2S. Especies y subespecies de aves de Colombia amenazadas en Negret 2001. Los nombres de las especies están actualizados de acuerdo con SACC (2016), pero se conserva la clasificación original de las familias de ese libro. Vea la tabla $2 \mathrm{~S}$ en: https://www.raccefyn. co/index.php/raccefyn/article/downloadSuppFile/461/2389

Tabla 3S. Disponibilidad de información para obtener estimaciones de tamaño poblacional de las especies amenazadas en Renjifo, et al. 2014 y Renjifo, et al. 2016. Vea la tabla 3S en: https:/www.raccefyn. co/index.php/raccefyn/article/downloadSuppFile/461/2390

Tabla 4S. Proporción de amenaza por familia de las aves residentes permanente o estacionalmente en Colombia. Vea la tabla 4S en: https://www.raccefyn.co/index.php/raccefyn/article/download SuppFile/461/2391

\section{Conflicto de intereses}

Los autores declaran que no tienen conflicto de intereses.

\section{Contribución de los autores}

Los dos autores de este artículo contribuyeron igualmente en la compilación, revisión y síntesis de la información, el análisis de resultados, la discusión y la escritura del manuscrito.

\section{Referencias}

Amat, G.G., Andrade, M.G., Amat, G.E.C. (2007). Libro rojo de especies amenazadas de invertebrados terrestres de Colombia. Conservación Internacional Colombia, Instituto de Ciencias Naturales de Colombia, Instituto de Investigación de Recursos Biológicos Alexander von Humboldt, Ministerio de Ambiente, Vivienda y Desarrollo Territorial. Bogotá, Colombia.

Amaya-Espinel, J.D, Gómez, M.F., Amaya-Villarreal, A.M., Velásquez Tibatá, J., Renjifo L.M. (2011). Guía metodológica para el análisis de riesgo de extinción de especies en Colombia. Ministerio de Ambiente, Vivienda y Desarrollo Territorial, Instituto de Investigación de Recursos Biológicos Alexander von Humboldt y Pontificia Universidad Javeriana. p. 84.

Arboleda, N. (2008). La palma africana en el Pacífico colombiano: su ilegalidad, consecuencias y violación de derechos territoriales. Revista Luna Azul. 27: 113-126.

Ardila, N., Navas, G., Reyes, J. (eds.). (2002). Libro rojo de invertebrados marinos de Colombia. Serie Libros Rojos de Especies Amenazadas de Colombia. Invemar y Ministerio del Medio Ambiente. Santa Marta, Colombia.

Avendaño, J.E., Cuervo, A.M., López, J.P., Gutiérrez-Pinto, N., Cortés-Diago, A., Cadena, C.D. (2015). A new species of tapaculo (Rhinocryptidae: Scytalopus) from the Serranía de Perijá of Colombia and Venezuela. The Auk. 132: 450-466. 
Ayerbe-Quiñones, F. (2016). Cypseloides lemosi. En: Renjifo, L. M., Amaya-Villarreal A. M., Burbano-Girón, J., VelásquezTibatá, J. 2016. Libro rojo de aves de Colombia, Volumen II: Ecosistemas abiertos, secos, insulares, acuáticos continentales, marinos, tierras altas del Darién y Sierra Nevada de Santa Marta y bosques húmedos del centro, norte y oriente del país. Editorial Pontificia Universidad Javeriana e Instituto Alexander von Humboldt. Bogotá, D. C., Colombia. p. 463-465.

Ayerbe-Quiñones, F., Pulgarín-Restrepo, P., Estela, F.A. (2016). Podiceps occipitalis. En: Renjifo, L. M., Amaya-Villarreal A. M., Burbano-Girón, J., Velásquez-Tibatá, J. (2016). Libro rojo de aves de Colombia, Volumen II: Ecosistemas abiertos, secos, insulares, acuáticos continentales, marinos, tierras altas del Darién y Sierra Nevada de Santa Marta y bosques húmedos del centro, norte y oriente del país. Editorial Pontificia Universidad Javeriana e Instituto Alexander von Humboldt. Bogotá, D. C., Colombia. p. 119-122.

Banco Mundial (2006). Fortalecimiento de la gobernabilidad y aplicación de la legislación forestal: confrontando un obstáculo sistémico al desarrollo sostenible. Washington, EUA.

BirdLife International. (2017). Country profile: Colombia. Fecha de consulta: 21 de febrero de 2017. Disponible en: http:// www.birdlife.org/datazone/countrycolombia. Checked: 201702-21

Böhm, M., Collen, B., Baillie, J.E., Bowles, P., Chanson, J., Cox, N., Zug, G. (2013). The conservation status of the world's reptiles. Biological Conservation. 157: 372-385.

Bravo, G.A., Naranjo, L. G. (2006). Estado del conocimiento sobre aves terrestre en Colombia. En: M.E. Chaves y M. Santamaría (eds.). Informe nacional sobre el estado de avance en el conocimiento y la información de la biodiversidad 1998-2004. Tomo 2. Instituto de Investigación de Recursos Biológicos Alexander von Humboldt. Bogotá. p. $130-150$.

Brooks, D.M., Cancino, L., Pereira, S.L. (2006). Conserving cracids: The most threatened family of birds in the Americas. Miscellaneous Publications of the Houston Museum of Natural Science. 6.

Cabrera, E., Vargas, D.M., Galindo, G., García, M.C., Ordóñez, M.F., Vergara, L.K., Giraldo, P. (2011). Memoria técnica de la cuantificación de la deforestación histórica nacionalescalas gruesa y fina. Instituto de Hidrología, Meteorología y Estudios Ambientales-IDEAM, Bogotá, Colombia.

Calderón-Sáenz, E. (Ed.). (2007). Libro rojo de plantas de Colombia. Volumen 6: Orquídeas. Primera Parte. Serie Libros Rojos de Especies Amenazadas de Colombia. Ministerio de Ambiente, Vivienda y Desarrollo Territorial e Instituto de Investigación de Recursos Biológicos Alexander von Humboldt. Bogotá, Colombia.

Calderón, E., Galeano, G., García, N. (Eds.). (2002). Libro rojo de plantas fanerógamas de Colombia. Volumen 1: Chrysobalanaceae, Dichapetalaceae y Lecythidaceae. La serie Libros rojos de especies amenazadas de Colombia. Bogotá, Colombia. Instituto Alexander von Humboldt, Instituto de Ciencias Naturales-Universidad Nacional de Colombia, Ministerio del Medio Ambiente.

Calderón, E., Galeano, G., García, N. (Eds.). (2005). Libro rojo de plantas de Colombia. Volumen 2: Palmas, frailejones y zamias. Serie Libros rojos de especies amenazadas de
Colombia. Instituto de Investigación de Recursos Biológicos Alexander von Humboldt, Instituto de Ciencias Naturales-Universidad Nacional de Colombia, Ministerio del Medio Ambiente. Bogotá, Colombia.

Carantón-Ayala, D., Certuche-Cubillos, K. (2010). A New Species of Antpitta (Grallariidae: Grallaria) from the Northern Sector of the Western Andes of Colombia. Ornitología Colombiana. 9: 56-70.

Cárdenas, L.D., Salinas, N.R. (Eds.). (2007). Libro rojo de plantas de Colombia. Volumen 4. Especies maderables amenazadas: Primera parte. Serie Libros rojos de especies amenazadas de Colombia. Bogotá, Colombia. Instituto Amazónico de Investigaciones Científicas Sinchi y Ministerio de Ambiente, Vivienda y Desarrollo Territorial. p. 232.

Carson, R. (1962). Silent Spring. Houghton-Mifflin, Boston, USA.

Castaño-Mora, O. V. (Ed.). (2002). Libro rojo de reptiles de Colombia. Serie Libros Rojos de Especies Amenazadas de Colombia. Instituto de Ciencias Naturales-Universidad Nacional de Colombia, Ministerio del Medio Ambiente, Conservación Internacional Colombia. Bogotá, Colombia.

Chasqui V., Polanco, L.A., Acero, F.A., Mejía-Falla, P.A., Navia, A., Zapata, L.A., Caldas, J.P. (Eds.) (2017). Libro rojo de peces marinos de Colombia. Instituto de Investigaciones Marinas y Costeras Invemar, Ministerio de Ambiente y Desarrollo Sostenible. Serie de Publicaciones Generales de INVEMAR \# 93. Santa Marta, Colombia.

Clausnitzer, V., Kalkman, V.J., Ram, M., Collen, B., Baillie, J.E., Bedjanič, M., Karube, H. (2009). Odonata enter the biodiversity crisis debate: The first global assessment of an insect group. Biological Conservation. 142 (8): 1864-1869.

Collen, B., Loh, J., Whitmee, S., McRae, L. Amin, R., Baillie, J. E. (2009). Monitoring change in vertebrate abundance: The Living Planet Index. Conservation Biology. 23 (2): 317-327.

Contraloría General de la República. (2012). Cerca del 80\% de la minería en Colombia es ilegal, advierte la Contraloría. Fecha de consulta: 21 de febrero de 2017. Disponible en: http://www.lafm.com.co/noticias/colombia/05-11-12/ cerca-del-80-de-la-mineria-en--12

Cortés-Diago, A., Ortega, L.A., Mazariegos-Hurtado, L., Weller, A. A. (2007). A new species of Eriocnemis (Trochilidae) from southwest Colombia. Ornitología Neotropical. 18 (2): 161-170.

Cuervo, A.M., Salaman, P.G.W., Donegan, T.M., Ochoa, J.M. (2001). Una nueva especie de Piha (Cotingidae: Lipaugus) de la Cordillera Central de Colombia. Ibis. 143: 353-368.

Cuervo, A.M., Cadena, C.D., Krabbe, N., Renjifo, L.M. (2005). Scytalopus stilesi, a new species of tapaculo (Rhinocryptidae) from the Cordillera Central of Colombia. The Auk. 122 (2): 445-463.

Dávalos, L.M., Bejarano, A.C., Correa, H.L. (2009). Disabusing cocaine: Pervasive myths and enduring realities of a globalised commodity. International Journal of Drug Policy. 20 (5): 381-386.

Dávalos, L.M., Bejarano, A.C., Hall, M.A., Correa, H.L., Corthals, A., Espejo, O.J. (2011). Forests and drugs: coca-driven deforestation in tropical biodiversity hotspots. Environmental science \& technology. 45 (4): 1219-1227.

De las Casas, J. C. (2004). Evaluación del estado taxonómico del Semillero de Tumaco Sporophila insulata (Fringillidae: Emberizinae) utilizando métodos morfológicos y genéticos. Tesis de pregrado. Universidad Nacional de Colombia, Bogotá, Colombia. 
Dion, M.L., Russler, C. (2008). Eradication efforts, the state, displacement and poverty: Explaining coca cultivation in Colombia during Plan Colombia. Journal of Latin American Studies. 40: 399-421.

Dirzo, R., Young, H.S., Galetti, M., Ceballos, G., Isaac, N.J., Collen, B. (2014). Defaunation in the Anthropocene. Science. 345 (6195): 401-406.

Donegan, T. M. (2007). A new species of brush finch (Emberizidae: Atlapetes) from the northern Central Andes of Colombia. Bulletin of the British Ornithologists' Club. 127 (4): 255-268.

EJOLT Project. (2016). Environmental Justice Organizations, Liabilities and Trade. Fecha de consulta: 21 de febrero de 2017. Disponible en: http://ejatlas.org/country/colombia

Estela, F. A., López-Victoria, M., Castillo, L. F., Naranjo, L. G. (2010). Estado del conocimiento sobre aves marinas en Colombia, después de 110 años de investigación. Boletín são. 20: 2-21.

Estes, J. A., Brashares, J. S., Power, M. E. (2013). Predicting and detecting reciprocity between indirect ecological interactions and evolution. The American Naturalist. 181 (S1): S76-S99.

Etter, A. (1993). Diversidad ecosistémica en Colombia hoy. En: Cárdenas S. y M. D. Correa (eds). Nuestra diversidad biológica. Fundación Alejandro Ángel Escobar. Bogotá. p. 43-61

Kellman, M. (1989). Mineral nutrient dynamics during savanna forest transformation in Central America. Mineral nutrients in p. 43-61.

Etter, A. (1998). Mapa general de ecosistemas de Colombia. En M. E. Cháves y N. Arango, editores. Informe nacional sobre el estado de la biodiversidad. Colombia 1997. Tomo 1. Diversidad biológica. Instituto de Investigación de Recursos Biológicos Alexander von Humboldt, PNUMA, Ministerio del Medio Ambiente, Bogotá D. C., Colombia. p. 1-150.

Etter, A., McAlpine, C., Pullar, D., Possingham, H. (2006). Modelling the conversion of Colombian lowland ecosystems since 1940: Drivers, patterns and rates. Journal of Environmental Management. 79: 74-87.

Etter, A., Andrade A., Amaya, P., Arévalo, P. (2015). Estado de los ecosistemas colombianos- 2014: una aplicación de la metodología de lista roja de ecosistemas. Fecha de consulta: 20 marzo 2017. Disponible en: https://iucnrle.org/static/ media/uploads/references/published-assessments/etteretal-2015-national-rle-assessment-final-report-colombiasp.pdf

Farrier, D., Whelan, R., Mooney, C. (2007). Threatened species listing as a trigger for conservation action. Environmental Science \& Policy. 10: 219-229.

Foley, J.A., DeFries, R., Asner, G.P., Barford, C., Bonan, G., Carpenter, S. R., Helkowski, J.H. (2005). Global consequences of land use. Science. 309 (5734): 570-574.

Franco, A.M., Devenish, C., Barrero, M.C., Romero, M.H. (2009). Colombia. En: C. Devenish, D. F. Díaz Fernández, R. P. Clay, I. Davidson \& I. Y. Zabala (Eds). Important bird areas Americas-priority sites for biodiversity conservation. BirdLife International, Quito, Ecuador. p. 135-148.

Fundación Pro-Sierra Nevada de Santa Marta. (1998). Evaluación ecológica rápida: definición de áreas críticas para la conservación en la Sierra Nevada de Santa Marta, Bogotá D. C., Colombia.

García, H. (2014). Deforestación en Colombia: retos y perspectivas. Fedesarrollo. Fecha de consulta: 19 de abril de 2017. Disponible en: http://hdl.handle.net/11445/337
García, N., Galeano, G. (eds.). (2006). Libro rojo de plantas de Colombia. Volumen 3: Las bromelias, las labiadas y las pasifloras. Instituto de Investigación de Recursos Biológicos Alexander von Humboldt, Instituto de Ciencias Naturales-Universidad Nacional de Colombia y Ministerio del Medio Ambiente. Bogotá, Colombia.

García, N. (ed.). (2007). Libro rojo de plantas de Colombia. Volumen 5: Las magnoliáceaes, las miristicáceaes y las podocarpáceaes. Serie Libros rojos de especies amenazadas de Colombia. Instituto de Investigación de Recursos Biológicos Alexander von Humboldt, Corantioquia, Jardín Botánico Joaquín Antonio Uribe de Medellín, Instituto de Ciencias Naturales - Universidad Nacional de Colombia y Ministerio de Ambiente, Vivienda y Desarrollo Territorial. Bogotá, Colombia.

Garnett, S. T., Christidis, L. (2017). Taxonomy anarchy hampers conservation. Nature. 546: 25-27.

Gill, F., Donsker, D. (eds). (2017). IOC World Bird List (v 7.1). https://doi.org:10.14344/IOC.ML.7.1. Fecha de consulta: 23 de enero 2017. Disponible en: http://www. worldbirdnames.org/

Gregory, R.D., van Strien, A. (2010). Wild bird indicators: Using composite population trends of birds as measures of environmental health. Ornithology Science. 9: 3-22.

Haddad, N.M., Brudvig, L. A., Clobert, J., Davies, K. F., González, A., Holt, R.D., Townshend, J. R. (2015). Habitat fragmentation and its lasting impact on Earth's ecosystems. Science Advances. 1 (2): e1500052. https://doi. org.10.1126/sciadv.1500052

Hilty, S. L. (1985). Distributional changes in the Colombian avifauna: A preliminary Blue List. En, P. A. Buckley, M. S. Foster, E. S. Morton, R. S. Ridgely, y F. G. Buckley, (Eds). Neotropical Ornithology. Ornithological Monographs No. 36. American Ornithologists'Union. Lawrence, Kansas, USA. p. 1000-1012.

Hilty, S. L., Brown, W.L. (1986). A guide to the birds of Colombia. Princeton University Press. Princeton, N.J., USA.

Hoffmann, M., Hilton-Taylor, C., Angulo, A., Böhm, M., Brooks, T. M., Butchart, S.H., Darwall, W.R. (2010). The impact of conservation on the status of the world's vertebrates. Science. 330 (6010): 1503-1509.

Isaac, N.J., Mallet, J., Mace, G.M. (2004). Taxonomic inflation: Its influence on macroecology and conservation. Trends in Ecology \& Evolution. 19 (9): 464-469.

IUCN. (2001). IUCN Red List Categories and Criteria: Version 3.1. Gland, Switzerland and Cambridge, UK.

IUCN. (2012). Guidelines for Application of IUCN Red List Criteria at Regional and National Levels: Version 4.0. Gland, Switzerland and Cambridge, UK: IUCN. p. iii +41 .

IUCN. (2015). IUCN Red List of Threatened Species. Version 2015.2. Fecha de consulta: 4 de junio de 2015. Disponible en: www.iucnredlist.org.

Keith, D.A., Rodríguez, J.P., Rodríguez-Clark, K.M., Aapala, K., Alonso A., Asmussen, M., Zambrano-Martínez, S. (2013). Scientific Foundations for an IUCN Red List of Ecosystems. PLoS ONE. 8 (5): e62111.

Kellman, M. (1989). Mineral nutrient dynamics during savanna forest transformation in Central America. En: Mineral Nutrients in Tropical Forest and Savanna Ecosystems. Blackwell, Oxford, UK. 
Kirchman, J.J., Witt, C.C., McGuire, J.A., Graves, G.R. (2009). DNA from a 100-year-old holotype confirms the validity of a potentially extinct hummingbird species. Biology Letters. 6: $112-115$.

Krabbe, N., Salaman, P., Cortés, A. Quevedo, A., Ortega, L. A., Cadena, C.D. (2005a). A new species of tapaculo from the upper Magdalena valley, Colombia. Bulletin of the British Ornithologists' Club. 125: 93-108.

Krabbe, N., Flórez, P., Suárez, G., Castaño, J. (2005b). Rediscovery of the Dusky Starfrontlet Coeligena orina, with a description of the adult plumages and a reassessment of its taxonomic status. Ornitología Colombiana. 1: 28-35.

Lara, C. E., Cuervo, A. M., Valderrama, S.V., Calderón-F., D., Cadena, C. D. (2012). A new species of wren (Troglodytidae: Thryophilus) from the dry Cauca River Canyon, northwestern Colombia. The Auk. 129 (3): 537-550.

Laverde, O. (2014). Crypturellus obsoletus castaneus. En: Renjifo, L. M., Amaya-Villarreal A. M., Burbano-Girón, J. \& Velásquez-Tibatá, J. 2016. Libro rojo de aves de Colombia, Volumen II: Ecosistemas abiertos, secos, insulares, acuáticos continentales, marinos, tierras altas del Darién y Sierra Nevada de Santa Marta y bosques húmedos del centro, norte y oriente del país. Editorial Pontificia Universidad Javeriana e Instituto Alexander von Humboldt. Bogotá, D. C., Colombia.

Linares, E. L., Uribe-Meléndez, J. (2002). Libro rojo de briófitas de Colombia. Serie Libros Rojos de Especies Amenazadas de Colombia. Instituto de Ciencias Naturales Universidad Nacional de Colombia, Ministerio del Medio Ambiente. Bogotá, Colombia.

Loh, J., Wackernagel, M. (2004). Living planet report 2004. Fecha de consulta: 12 de julio de 2017. Disponible en: http://hdl.handle.net/10919/65909

Loh, J., Green, R. E., Ricketts, T., Lamoreux, J., Jenkins, M., Kapos, V., Randers, J. (2005). The Living Planet Index: Using species population time series to track trends in biodiversity. Philosophical Transactions of the Royal Society of London B: Biological Sciences. 360 (1454): 289-295.

Lozano-Jaramillo, M., Rico-Guevara, A., Cadena, C. D. (2014). Genetic Differentiation, Niche Divergence, and Origin and Maintenance of the Disjunct Distribution in the Blossomcrown Anthocephala floriceps (Trochilidae). PLoS ONE. 9 (9):e108345.

Mace, G. M., Lande, R. (1991). Assessing extinction threats: toward a reevaluation of threatened species categories. Conservation Biology. 5: 148-157.

Mace, G.M., Collar, N.J., Gaston, K.J., Hilton-Taylor, C., Akçakaya, H.R., Leader-Williams, N., Milner-Gulland, E.J., Stuart, S.N. (2008). Quantification of extinction risk: IUCN's system for classifying threatened species. Conservation Biology. 22: 1424-1442. https://doi.org.10. 1111/j.1523-1739.2008.01044.x

Mejía, L.S., Acero, A. (Eds.). (2002). Libro rojo de peces marinos de Colombia. INVEMAR, Instituto de Ciencias NaturalesUniversidad Nacional de Colombia, Ministerio de Medio Ambiente. La serie Libros rojo de especies amenazadas de Colombia. Bogotá, Colombia.

Mojica, J. I., Castellanos, C., Usma, S. y Álvarez, R. (Eds.). (2002). Libro rojo de peces dulceacuícolas de Colombia. Serie libros rojos de especies amenazadas de Colombia. Instituto de Ciencias Naturales- Universidad Nacional de Colombia y Ministerio del Medio Ambiente. Bogotá, Colombia.
Mojica, J.I., Usma, J.S. Álvarez-León, R., Lasso C.A. (Eds). (2012). Libro rojo de peces dulceacuícolas de Colombia. Instituto de Investigación de Recursos Biológicos Alexander von Humboldt, Instituto de Ciencias Naturales de la Universidad Nacional de Colombia, WWF Colombia y Universidad de Manizales. Bogotá, D. C., Colombia.

Naranjo, L.G., Bravo G.A. 2006. Estado del conocimiento sobre aves acuáticas en Colombia. En: M. E. Chaves y M. Santamaría (eds.). Informe nacional sobre el estado de avance en el conocimiento y la información de la biodiversidad 1998-2004. Tomo 2. Instituto de Investigación de Recursos Biológicos Alexander von Humboldt, Bogotá. p. 214-224.

Negret, A. J. (2001). Aves en Colombia amenazadas de extinción. Editorial Universidad del Cauca, Popayán, Colombia.

Ocampo-Peñuela, N. (2010). El fenómeno de la migración en aves: una mirada desde la Orinoquia. Orinoquia. 14: 188-200.

Pacheco-Garzón, A. (2012). Study and conservation of the birds of San Andrés island. Conservación Colombiana. 16: 5-54.

Parr M., Juniper T. (1998). Parrots: A Guide to Parrots of the World. Yale University Press, New Haven.

Parra, J.L. (2016). Heliangelus zusii. En: Renjifo, L. M., AmayaVillarreal A. M., Burbano-Girón, J. y Velásquez-Tibatá, J., (2016). Libro rojo de aves de Colombia, Volumen II: Ecosistemas abiertos, secos, insulares, acuáticos continentales, marinos, tierras altas del Darién y Sierra Nevada de Santa Marta y bosques húmedos del centro, norte y oriente del país. Editorial Pontificia Universidad Javeriana e Instituto Alexander von Humboldt. Bogotá, D. C., Colombia. p. 192-194

Pereira, H. M., Leadley, P. W, Proenca, V., Alkemade, R., Scharlemann, J. P. W., Fernández-Manjarres, J. F., Walpole, M. (2010). Scenarios for global biodiversity in the 21st century. Science. 330: 1496-1501.

Pérez-Emán, J.L., Ferreira, J.P., Gutiérrez-Pinto, N., Cuervo, A.M., Céspedes, L.N., Witt, C.C., Cadena, C.D. (2017) An extinct hummingbird species that never was a cautionary tale about sampling issues in molecular phylogenetics. bioR. Xiv: 149898; https://doi.org/10.1101/149898

Pérez-Rincón, M. (2014). Conflictos ambientales en Colombia: inventario, caracterización y análisis. En L. J. Garay (ed.). Minería en Colombia: control público, memoria y justicia socio-ecológica, movimientos sociales y pos-conflicto. Contraloría General de la República, Bogotá. 2014. p. 253325. Fecha de consulta: 19 de abril de 2017. Disponible en: https://redjusticiaambientalcolombia.files.wordpress. com/2014/08/libro-mineria_contraloria_vol-iv.pdf

Pimm, S. L., Raven, P. (2000). Biodiversity: extinction by numbers. Nature. 403 (6772), 843-845.

Pitman, R.L., Jehl Jr, J.R. (1998). Geographic variation and reassessment of species limits in the" masked” boobies of the eastern Pacific Ocean. The Wilson Journal of Ornithology. 110 (2): 154.

PNUD. (2011). Colombia rural. Razones para la esperanza. INDH PNUD. Bogotá, D.C, Colombia.

Proença, V., Martin, L. J., Pereira, H. M., Fernandez, M., McRae, L., Belnap, J., Honrado, J. P. (2017). Global biodiversity monitoring: from data sources to essential biodiversity variables. Biological Conservation. 213 (B): 256-263.

R Development Core Team. 2011. R: A Language and Environment for Statistical Computing. R Foundation for Statistical Computing, Vienna, Austria. 
Rands, M. R. W., Adams, W. M., Bennun, L., Butchart, S.H.M., Clements, A., Coomes, D., Entwistle, A., Hodge, I., Kapos, V., Scharlemann, J. P. W., Sutherland, W. J., Vira, B. (2010). Biodiversity conservation: challenges beyond. Science. 329: 1298-1303.

Remsen J.V. Jr., Areta J.I., Cadena, C.D., Claramunt, S., Jaramillo, A., Pacheco, J. F., Robbins M. B., Schulenberg, T. S., Stiles G., Stotz, D.F., Zimmer, K.J. (2016). A classification of the bird species of South America. American Ornithologists' Union, Washigton DC, USA. Fecha de consulta: 23 de agosto 2016. Disponible en: http://www. museum.lsu.edu/

Renjifo L.M., Amaya-Villarreal, A.M., Velásquez-Tibatá, Burbano-Girón J. (2016). Libro rojo de aves de Colombia. Volumen II: Ecosistemas abiertos, secos, insulares, acuáticos continentales, marinos, tierras altas del Darién y Sierra Nevada de Santa Marta y bosques húmedos del centro, norte y oriente del país. Editorial Pontificia Universidad Javeriana e Instituto Alexander von Humboldt. Bogotá, D.C. Colombia. (v. 2).

Renjifo L.M., Gómez, M.F., Velásquez-Tibatá, J., Kattan, G. H. Amaya-Espinel, J. D., Amaya-Villarreal, A.M., BurbanoGirón J. (2014). Libro rojo de aves de Colombia. Volumen I: Bosques húmedos de los Andes y la costa pacífica. Editorial Pontificia Universidad Javeriana e Instituto Alexander von Humboldt. Bogotá, D.C. Colombia ISBN 978-958-7166712 (v.1).

Renjifo, L. M. (1999). Composition Changes in a Subandean Avifauna after Long-Term Forest Fragmentation. Conservation biology. 13 (5): 1124-1139.

Renjifo, L. M. (1998). Especies de aves amenazadas y casi amenazadas de extinción en Colombia. Informe nacional sobre el estado de la biodiversidad Colombia, Tomo I. Diversidad biológica. Instituto de Investigación de Recursos Biológicos Alexander von Humboldt - Ministerio del Medio Ambiente - Programa de las Naciones Unidas para el Medio Ambiente (PNUMA).

Renjifo, L.M., Franco-Maya B., A.M., Amaya-Espinel, J.D., Kattan G.H., López-Lanus, B. (eds.). (2002). Libro rojo de aves de Colombia. Serie Libros rojos de especies amenazadas de Colombia. Instituto de Investigación de Recursos Biológicos Alexander von Humboldt y Ministerio del Medio Ambiente. Bogotá, Colombia. ISBN 958-8151-08-2.

Rincón Ruiz, A. R., Pascual, U., Romero, M. (2013). An exploratory spatial analysis of illegal coca cultivation in Colombia using local indicators of spatial association and socioecological variables. Ecological indicators. 34: 103-112.

Ripple, W. J., Estes, J. A., Beschta, R. L., Wilmers, C. C., Ritchie, E. G., Hebblewhite, M., Schmitz, O. J. (2014). Status and ecological effects of the world's largest carnivores. Science. 343 (6167): 1241484.

Rodrigues, A., Pilgrim, J. D., Lamoureux, J. F., Hoffman, M., Brooks, T. M. (2006). The value of the IUCN Red List for conservation. Trends in Ecology and Evolution. 21 (2): 71-76.

Rodríguez-Mahecha, J. V., Alberico, M., Trujillo, F., Jorgenson, J. (Eds.). 2006. Libro rojo de los mamíferos de Colombia. Serie libros rojos de especies amenazadas de Colombia. Conservación Internacional Colombia y Ministerio de Ambiente, Vivienda y Desarrollo Territorial. Bogotá, Colombia.
Rodríguez-Mahecha, J.V., Hernández-Camacho, J.I., Rueda, J.V., Morales J. (1986). Colombia, fauna en peligro. (1986). Revista Procam - Inderena. 1: 1-17.

Rodríguez, J.P., Balch, J.K., Rodríguez-Clark, K. M. (2007). Assessing extinction risk in the absence of species-level data: Quantitative criteria for terrestrial ecosystems. Biodiversity Conservation. 16: 183-209.

Rojas-Briñez, D.K.R., Silva, M.R., Melo, J.E.G. (2013). Estado actual y perspectivas de conservación frente al comercio ilegal de fauna silvestre en el departamento del Tolima (Colombia). Revista Tumbaga. p. 97-111.

Rueda-Almonacid, J.V., Lynch, J.D., Amézquita, A. (eds.). 2004. Libro rojo de anfibios de Colombia. Serie Libros rojos de especies amenazadas de Colombia. Conservación Internacional, Instituto de Ciencias Naturales-Universidad Nacional de Colombia, Ministerio del Medio Ambiente. Bogotá, Colombia.

Salafsky, N., Salzer, D., Stattersfield, A. J., Hilton-Taylor, C., Neugarten, R., Butchart, S.H., Collen, B., Cox, N., Master, L.L., O'Connor, S., Wilkie, D. (2008). A standard lexicon for biodiversity conservation: Unified classifications of threats and actions. Conservation Biology. 22 (4): 897-911.

Salaman, P., Coopmans, P., Donegan, T.M., Mulligan, M., Cortés, A., Hilty S.L., Ortega L.A. (2003). A new species of woodwren (Troglodytidae: Henicorhina) from the western Andes of Colombia. Ornitología Colombiana. 1: 4-21.

Sánchez-Cuervo, A.M., Aide, T.M., Clark, M.L., Etter, A. (2012). Land cover change in Colombia: Surprising forest recovery trends between 2001 and 2010. PLoS One. 7: 1-14.

Schipper, J., Chanson, J.S., Chiozza, F., Cox, N.A., Hoffmann, M., Katariya, V., Baillie, J. (2008). The status of the world's land and marine mammals: Diversity, threat, and knowledge. Science. 322 (5899): 225-230.

Şekercioğlu, C.H. (2006). Increasing awareness of avian ecological function. Trends in ecology \& evolution. 21 (8): 464-471.

Snyder, N., McGowan, P., Gilardi, J., Grajal, A. (Eds.). (2000). Parrots. Status Survey and Conservation Action Plan 20002004. International Union for the Conservation of Nature. Gland, Switzerland \& Cambridge, UK.

Stiles, F.G. 2004. The Tumaco Seedeater (Sporophila insulata, Emberizidae): A species that never was? Ornitologia Neotropical. 15: 17-30.

Sokal, R.R., Rohlf F.J. (1995). Biometry: The principles and practice of statistics in biological research. $3^{\text {rd }}$ Edition. W.H. Freeman and Company, USA.

Stattersfield, A.J., Crosby, M.J., Long, A.J., Wege, D. (1998). Endemic bird areas of the world. Priorities for biodiversity conservation. BirdLife International, Cambridge, UK.

UICN. (2003). Directrices para emplear los criterios de la Lista Roja de la UICN a nivel regional: Versión 3.0. Comisión de Supervivencia de Especies de la UICN. UICN, Gland, Suiza y Cambridge, Reino Unido.

Wenny, D. G., Devault, T.L., Johnson, M.D., Kelly, D., Şekercioğlu, C.H., Tomback, D.F., Whelan, C.J. The Need to Quantify Ecosystem Services Provided By Birds. The Auk. 128 (1): 1-14.

Whelan, C.J., Şekercioğlu, Ç.H., Wenny, D.G. (2015). Why birds matter: From economic ornithology to ecosystem services. Journal of Ornithology. 156: 227-238. 